\title{
Uncovering the potential differentially expressed miRNAs as diagnostic biomarkers for hepatocellular carcinoma based on machine learning in The Cancer Genome Atlas database
}

\author{
XIN ZHAO ${ }^{1}$, JIAN DOU ${ }^{1}$, JINGLIN CAO ${ }^{1}$, YANG WANG ${ }^{1}$, QINGJUN GAO ${ }^{1}$, QIANG ZENG ${ }^{1}$, WENPENG LIU ${ }^{1}$, \\ BAOWANG LIU ${ }^{1}$, ZIQIANG CUI ${ }^{1}$, LIANG TENG ${ }^{1}$, JUNHONG ZHANG $^{1}$ and CAIYAN ZHAO ${ }^{2}$ \\ Departments of ${ }^{1}$ Hepatobiliary Surgery and ${ }^{2}$ Infection, \\ The Third Hospital of Hebei Medical University, Shijiazhuang, Hebei 050000, P.R. China
}

Received September 11, 2019; Accepted January 22, 2020

DOI: 10.3892/or.2020.7551

\begin{abstract}
The present study aimed to identify novel diagnostic differentially expressed microRNAs (miRNAs/miRs) in order to understand the molecular mechanisms underlying hepatocellular carcinoma. The expression data of miRNA and mRNA were downloaded for differential expression analysis. Optimal diagnostic differentially expressed miRNA biomarkers were identified via a random forest algorithm. Classification models were established to distinguish patients with hepatocellular carcinoma and normal individuals. A regulatory network between optimal diagnostic differentially expressed miRNA and differentially expressed mRNAs was then constructed. The GSE63046 dataset and in vitro experiments were used to validate the expression of the optimal diagnostic differentially expressed miRNAs identified. In addition, diagnostic and prognostic analyses of optimal diagnostic differentially expressed miRNAs were performed. In total, 14 differentially expressed miRNAs (all upregulated) and 2,982 differentially expressed mRNAs (1,989 upregulated and 993 downregulated) were identified. hsa-miR-10b-5p, hsa-miR-10b-3p, hsa-miR-224-5p, hsa-miR-183-5p and
\end{abstract}

Correspondence to: Professor Caiyan Zhao, Department of Infection, The Third Hospital of Hebei Medical University, 68 Xiangjiang Road, Yuhua, Shijiazhuang, Hebei 050000, P.R. China E-mail: zhaocy2005@163.com

Abbreviations: DT, decision tree; EDNRB, endothelin receptor type B; FDR, false discovery rate; FOXO1, forkhead box O1; FHL2, four and a half LIM domains 2; GO, Gene Ontology; IL6ST, interleukin 6 signal transducer; KEGG, Kyoto Encyclopedia of Genes and Genomes; NKX3-1, NK3 homeobox 1; NR4A3, nuclear receptor subfamily 4 group A member 3 ; RF, random forests; ROC, receiver operating characteristic; SFRP1, secreted frizzled related protein 1; SVM, support vector machine

Key words: hepatocellular carcinoma, differentially expressed microRNAs, diagnosis, prognosis, machine learning
hsa-miR-182-5p were considered as the optimal diagnostic biomarkers for hepatocellular carcinoma. The mRNAs targeted by these five miRNAs included secreted frizzled related protein 1 (SFRP1), endothelin receptor type B (EDNRB), nuclear receptor subfamily 4 group A member 3 (NR4A3), four and a half LIM domains 2 (FHL2), NK3 homeobox 1 (NKX3-1), interleukin 6 signal transducer (IL6ST) and forkhead box O1 (FOXO1). 'Bile acid biosynthesis and cholesterol' was the most enriched signaling pathways of these target mRNAs. The expression validation of the five miRNAs was consistent with the present bioinformatics analysis. Notably, hsa-miR-10b-5p and hsa-miR-10b-3p had a significant prognosis value for patients with hepatocellular carcinoma. In conclusion, the five differentially expressed miRNAs may be considered as diagnostic biomarkers for patients with hepatocellular carcinoma. In addition, the differential expression levels of the targets of these five mRNAs, including SFRP1, EDNRB, NR4A3, FHL2, NKX3-1, IL6ST and FOXO1, may be involved in hepatocellular carcinoma tumorigenesis.

\section{Introduction}

Hepatocellular carcinoma is the third leading cause of cancer-associated mortality (1). The most significant characteristics of hepatocellular carcinoma are aggressiveness, invasiveness and frequent recurrence (2). Several risk factors for hepatocellular carcinoma have been identified, including liver cirrhosis, alcohol abuse, steatohepatitis, obesity, diabetes, intake of the fungal metabolite aflatoxin B1 and metabolic syndromes (3-8). In addition, frequent hyper-methylation of tumor suppressor genes, including p16, suppressor of cytokine signaling 1, glutathione S-transferase pi 1, Ras association (RalGDS/AF-6) domain family member $1 \mathrm{~A}$ and E-cadherin have been involved in the tumorigenesis of hepatocellular carcinoma (9-11). Clinically, surgical resection, interventional therapy, liver transplantation, liver-directed therapy and systemic therapy are common treatment methods (12). However, only liver transplantation and surgical resection are regarded as effective treatments. In addition, only $15 \%$ patients are eligible for effective treatments, whereas most patients present with advanced disease at diagnosis (13). Although 
some new therapeutic methods have been developed, the 5 -year survival rate of hepatocellular carcinoma remains poor due to late diagnosis, and the survival rate is currently $7 \%$ (14). Therefore, identifying biomarkers for the early diagnosis of hepatocellular carcinoma is required.

MicroRNAs (miRNAs/miRs) play a crucial role in regulating cell proliferation, differentiation, migration and apoptosis $(15,16)$. The expression of miRNAs has been previously investigated in hepatocellular carcinoma, and several miRNAs, such as hsa-miR-21, hsa-miR-223 and hsa-miR-122, were identified as upregulated $(17,18)$; while certain miRNAs, such as hsa-miR-122a, hsa-miR-152 and hsa-miR-22, were downregulated in hepatocellular carcinoma tissues (19-21). A previous study demonstrated that miRNAs may be used as biomarkers and therapeutic targets for the diagnosis and treatment of hepatocellular carcinoma (22). In the present study, in order to identify potential diagnostic biomarkers, differentially expressed miRNAs and mRNAs were investigated in hepatocellular carcinoma based on The Cancer Genome Atlas (TCGA) database. A machine learning approach was used to identify the differentially expressed miRNAs with diagnostic potential for hepatocellular carcinoma. Subsequent analysis was based on these differentially expressed miRNAs with diagnostic potential.

\section{Materials and methods}

Data retrieval. TCGA (http://tcga-data.nci.nih.gov/) is a publicly funded project which consists of multidimensional data of for multiple cancer types at DNA, RNA and protein levels. In the database, the clinical data of 377 patients, the mRNA data of 371 patients and the miRNA data of 373 patients were recorded. Details of the dataset are presented in Table SI. The mean age of these patients was $59.45 \pm 13.5$. In addition, the male:female ratio of the patients was $255: 122$. The clinical information of these patients in the TCGA is presented in Table SI. The expression data of miRNAs and mRNAs were generated using an RNA sequencing platform. Transcriptome mRNA and miRNA data of hepatocellular carcinoma were all obtained from primary solid tumors and normal solid tissues. Screening requirements for the included samples were as follows: i) Samples without clinical information were excluded; ii) samples with incomplete information about stage and survival time were excluded; and iii) samples with information about both miRNA and mRNA expression levels were reserved. According to the inclusion and exclusion criteria, the mRNA and miRNA data (including 342 cases and 50 controls) were finally used for the following integrated analysis.

Identification of differentially expressed miRNAs and $m R N A s$. Before identification, data of miRNAs and mRNAs were preprocessed, and miRNAs and mRNAs that were lowly expressed were deleted. miRNAs and mRNAs were considered to have low expression when the number of control samples presenting read counts value of 0 was $>20 \%$ of the total case sample size or when the number of case samples presenting read counts value of 0 was $>20 \%$ of the total control sample size. Principal component analysis of these miRNAs and mRNAs was subsequently conducted. Differentially expressed miRNAs and mRNAs were analyzed, as previously described (23). The false discovery rate (FDR) was obtained from multiple comparisons using The Benjamini and Hochberg method (24). Those differentially expressed miRNAs and mRNAs were identified with the criterion of FDR $<0.05$, abs $\mid\left(\log _{2}\right.$ FoldChange $) \mid>3$ and $F D R<0.05$, abs $\mid(\log 2$ FoldChange $) \mid>1$, respectively. In addition, the heat map of differentially expressed miRNAs and mRNAs was performed. Clustering was analyzed using the complete-linkage method together with the Euclidean distance.

Identification of the optimal diagnostic biomarkers based on a machine learning approach. Firstly, the importance value of each differentially expressed miRNA was ranked using a random forests ( $\mathrm{RF}$ ) algorithm. Then, the optimal number of features was identified by subsequently adding one differentially expressed miRNA at a time in a top down forward-wrapper approach. Optimal differentially expressed miRNA with diagnostic value for hepatocellular carcinoma were used to establish classification models, including RF, support vector machine (SVM) and decision tree (DT). The 'randomForests' package in R language (https://cran.r-project. org/web/packages/randomForest/), 'e1071' package in $\mathrm{R}$ language (https://cran.r-project.org/web/packages/e1071/index. html) and 'rpart' package in R language (https://cran.r-project. org/web/packages/rpart/index.html) were used to establish the RF model, SVM model and DT models, respectively. Diagnostic ability of classification prediction was evaluated by obtaining specificity, sensitivity and the area under a receiver operating characteristic (ROC) curve (AUC).

Network of differentially expressed miRNAs and mRNAs. The pairwise Pearson correlation coefficients between key differentially expressed miRNAs and mRNAs were calculated. In total, six miRNA-target prediction tools, including miRWalk (version 2.0; http://www.umm.uni-heidelberg. de/apps/zmf/mirwalk/index.html), miRanda (http://www. microrna.org/), miRDB(version 2.0; http://mirdb.org/miRDB/), RNA22 (version 2.0; https://cm.jefferson.edu/rna22v2.0/), PICTAR2 (version 2.0; http://pictar.mdc-berlin.de/) and Targetscan (version 6.2; http://www.targetscan.org/) were used to predict the genes targeted by the differentially expressed miRNAs. Subsets of miRNA-target pairs with negative correlations were used to establish the regulatory network using Cytoscape software (version 3.3.0) (25). In addition, concrete ATCG base binding sites in the identified miRNA-mRNA pairs were also detected based on the Starbase database (http://starbase.sysu.edu.cn/index.php).

Functional analysis of the target mRNAs. To understand the biological function of the target mRNAs, Gene Ontology (GO) (http://www.geneontology.org/) and Kyoto Encyclopedia of Genes and Genomes (KEGG; version 2.2.0; http://www.genome.jp/kegg/pathway.html) pathway enrichment analyses were performed using Metascape (version 3.3.0) (http://metascape.org/gp/index.html). $\mathrm{P}<0.05$ was considered to indicate a statistically significant difference.

In silico validation and in vitro validation. The Gene Expression Omnibus dataset GSE63046 (26) (involving 24 cases and 24 controls) was used to validate the expression 
Table I. A total of 14 differentially expressed miRNAs in hepatocellular carcinoma, which were all upregulated.

\begin{tabular}{llll}
\hline miRNA & $\log _{2}$ Fold Change & P-value & FDR \\
\hline hsa-miR-10b-5p & 3.603003957 & $1.14 \times 10^{-58}$ & $2.99 \times 10^{-56}$ \\
hsa-miR-224-5p & 3.343475173 & $1.70 \times 10^{-47}$ & $1.48 \times 10^{-45}$ \\
hsa-miR-183-5p & 3.870865325 & $3.51 \times 10^{-45}$ & $2.63 \times 10^{-43}$ \\
hsa-miR-1269a & 5.556896527 & $4.26 \times 10^{-42}$ & $2.79 \times 10^{-40}$ \\
hsa-miR-182-5p & 3.38187178 & $4.08 \times 10^{-38}$ & $2.14 \times 10^{-36}$ \\
hsa-miR-10b-3p & 3.549127719 & $1.34 \times 10^{-31}$ & $4.39 \times 10^{-30}$ \\
hsa-miR-96-5p & 3.726215737 & $2.36 \times 10^{-30}$ & $6.51 \times 10^{-29}$ \\
hsa-miR-217 & 4.036865911 & $2.25 \times 10^{-27}$ & $4.71 \times 10^{-26}$ \\
hsa-miR-9-5p & 3.161938401 & $2.98 \times 10^{-26}$ & $5.78 \times 10^{-25}$ \\
hsa-miR-196b-5p & 3.320156061 & $1.29 \times 10^{-25}$ & $2.25 \times 10^{-24}$ \\
hsa-miR-135a-5p & 4.141930478 & $8.47 \times 10^{-19}$ & $9.87 \times 10^{-18}$ \\
hsa-miR-216b-5p & 3.822995579 & $1.83 \times 10^{-18}$ & $2.05 \times 10^{-17}$ \\
hsa-miR-216a-5p & 3.427536419 & $7.89 \times 10^{-17}$ & $7.38 \times 10^{-16}$ \\
hsa-miR-552-5p & 3.736498894 & $6.81 \times 10^{-14}$ & $4.46 \times 10^{-13}$ \\
\end{tabular}

miRNA/miR, microRNA; FDR, false discovery rate.

of key differentially expressed miRNAs in tumor tissues compared with normal tissues from the same patients. The expression levels of these miRNAs are presented as box plots. Additionally, in vitro validation was performed by reverse transcription-quantitative PCR (RT-qPCR). Tumor and para-carcinoma tissues of seven patients were additionally collected for validation from December 30, 2018 to January 26, 2019 in The Third Hospital of Hebei Medical University. The clinical information (including therapy history, age and sex) of these patients was recorded before therapy.

The present study was approved by The Institutional Ethics Review Board of The Third Hospital of Hebei Medical University (approval no. 2018-025-1). In addition, informed consent was obtained from the individuals. Total RNA was extracted from tissue samples using TRIzol ${ }^{\circledR}$ (Invitrogen; Thermo Fisher Scientific, Inc.), according to the manufacturer's protocols. A total of $2 \mu \mathrm{g}$ RNA was used to synthesize cDNA using FastQuant Reverse Transcriptase (Sangon Biotech Co., Ltd.) for $60 \mathrm{~min}$ at $37^{\circ} \mathrm{C}$ followed by $5 \mathrm{~min}$ at $85^{\circ} \mathrm{C}$. qPCR was performed in an ABI 7300 Real-time PCR system with SYBR ${ }^{\circledR}$ Green PCR Master Mix (Applied Biosystems; Thermo Fisher Scientific, Inc.). The thermocycling conditions were as follows: Initial denaturation for $30 \mathrm{sec}$ at $95^{\circ} \mathrm{C}$ followed by 40 cycles of $5 \mathrm{sec}$ at $95^{\circ} \mathrm{C}$ and $30 \mathrm{sec}$ at $60^{\circ} \mathrm{C}$. All reactions were performed in triplicate. Hsa-U6 was used as the internal reference. The universal miRNA reverse primer is 5'-AACGAGACGACG ACAGAC-3'. The sequences of forward primers for all of the miRNAs analyzed were as follows: 5'-GCAAATTCGTGAAGC GTTCCATA-3' for Hsa-U6, 5'-UACCCUGUAGAACCGAAU UUGUG-3' for hsa-miR-10b-5p, 5'-ACAGAUUCGAUUCUA GGGGAAU-3' for hsa-miR-10b-3p, 5'-UCAAGUCACUAG UGGUUCCGUUUAG-3' for hsa-miR-224-5p, 5'-UAUGGC ACUGGUAGAAUUCACU-3' for hsa-miR-183-5p, and 5'-UUU GGCAAUGGUAGAACUCACACU-3' for hsa-miR-182-5p. The experiments were repeated three times. The relative gene expression levels were calculated as fold-changes using the $2^{-\Delta \Delta \mathrm{Cq}}$ method (27). The fold change was calculated as the enrichment between tumor tissue and para-carcinoma tissue.

In addition, according to the clinical information, patients with hepatocellular carcinoma were divided into two groups: i) Cirrhosis ( 75 cases); and ii) without cirrhosis (123 cases) to study whether liver cirrhosis may affect the expression of identified differentially expressed miRNAs.

Diagnosis and prognosis analysis of key differentially expressed miRNAs. ROC analysis was performed to assess the diagnostic value of key differentially expressed miRNAs. In addition, the survival package in $\mathrm{R}$ language (https://cran.r-project. org/web/packages/survival/index.html) was used to assess the prognostic value. The 5-year survival curves were plotted according to the clinical information and survival time.

Statistical analysis. All statistical analyses were performed using GraphPad Prism (version 8.0; GraphPad Software, Inc.). For the RT-qPCR experiments, one-way ANOVA, followed by Tukey's test to discriminate among the means, was used to assess statistical significance among two groups. For the box plots, the rank sum test was used to calculate the P-value. $\mathrm{P}<0.05$ was considered to indicate a statistically significant difference. Data are presented as the mean \pm SEM. All experiments were repeated independently at least three times.

\section{Results}

miRNA and mRNA expression pattern. First, principal component analyses for all miRNAs and mRNAs were performed. The present results demonstrated that these miRNAs and mRNAs were clearly separated according to the type of tissue, normal and tumor (Fig. S1). A total of 14 differentially expressed (all upregulated) miRNAs and 2,982 differentially expressed (1,989 upregulated and 993 downregulated) mRNAs 
Table II. Pairwise Pearson correlation analysis between five optimal differentially expressed miRNAs and their target differentially expressed mRNAs.

\begin{tabular}{lccc}
\hline mRNA & miRNA & cor & P-value \\
\hline SFRP1 & hsa-miR-10b-5p & -0.27623 & $2.70 \times 10^{-8}$ \\
EDNRB & hsa-miR-10b-3p & -0.14088 & 0.005201 \\
NR4A3 & hsa-miR-224-5p & -0.21914 & $1.20 \times 10^{-5}$ \\
NKX3-1 & hsa-miR-224-5p & -0.21888 & $1.23 \times 10^{-5}$ \\
FHL2 & hsa-miR-224-5p & -0.15065 & 0.002787 \\
IL6ST & hsa-miR-183-5p & -0.12462 & 0.013548 \\
FOXO1 & hsa-miR-182-5p & -0.20816 & $3.27 \times 10^{-5}$ \\
\hline
\end{tabular}

miRNA/miR, microRNA; cor, correlation coefficient; SFRP1, secreted frizzled related protein 1; EDNRB, endothelin receptor type B; $N R 4 A 3$, nuclear receptor subfamily 4 group A member 3 ; NKX3-1, NK3 homeobox 1; FHL2, four and a half LIM domains 2; $I L 6 S T$, interleukin 6 signal transducer; FOXO1, forkhead box $\mathrm{O} 1$.

were identified. The 14 differentially expressed miRNAs are presented in Table I. The heat maps corresponding to all miRNAs and top 50 mRNAs are presented in Figs. 1 and 2, respectively.

Identification of optimal diagnostic biomarkers based on a machine learning approach. The RF feature selection and classification (DT, SVM and RF) procedures were performed for the identification of diagnostic biomarkers. Alldifferentially expressed miRNAs were ranked according to the standardized drop in prediction accuracy (Fig. 3A). Differentially expressed miRNAs, including hsa-miR-10b-5p, hsa-miR-10b-3p, hsa-miR-224-5p, hsa-miR-183-5p and hsa-miR-182-5p, were considered as the optimal diagnostic biomarkers for hepatocellular carcinoma after subsequently adding one differentially expressed miRNA at a time in a top-down forward-wrapper approach (Fig. 3B). These five optimal differentially expressed miRNAs with diagnostic value for hepatocellular carcinoma were used to establish various classification models, including DT, SVM and RF. The AUC values in the RF, SVM and DT models were 98.2,97 and 83.1\%, respectively (Fig. 4). The RF model (with the largest AUC value) could effectively predict hepatocellular carcinoma.

Network of differentially expressed miRNAs and mRNAs. The correlation analysis between the five optimal differentially expressed miRNAs (hsa-miR-10b-5p, hsa-miR-10b-3p, hsa-miR-224-5p, hsa-miR-183-5p and hsa-miR-182-5p) and differentially expressed mRNAs was then performed. Following correlation analysis, 3,756 miRNA-mRNA pairs were identified to be negatively correlated $(\mathrm{P}<0.05 ; \mathrm{r}<0)$. In the target prediction and negative correlation analyses, 170 miRNA-mRNA pairs, including five miRNAs (upregulated) and 145 mRNA (downregulated) were identified. The established regulatory network of miRNA-targeted mRNAs with negative correlation is presented in Fig. 5. Pairwise Pearson correlation analyses between the five optimal differentially expressed miRNAs (hsa-miR-10b-5p, hsa-miR-10b-3p, hsa-miR-224-5p, hsa-miR-183-5p and hsa-miR-182-5p)

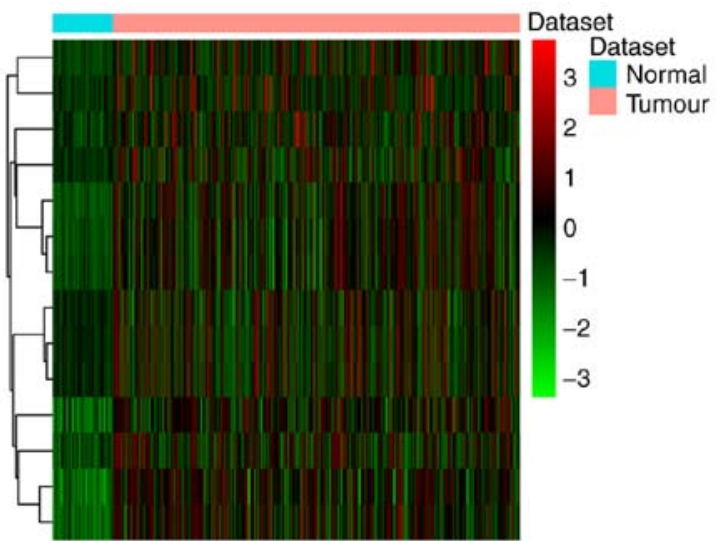

Figure 1. Heat map of all differentially expressed miRNAs in hepatocellular carcinoma. The diagram shows the result of a two-way hierarchical clustering of all differentially expressed miRNAs and samples. Clustering was analyzed using the complete-linkage method together with the Euclidean distance. Each row represents a differentially expressed miRNA and each column represents a sample. The differentially expressed miRNA color clustering tree is presented on the right. The color scale illustrates the relative expression level of differentially expressed miRNAs. Red indicates below the reference channel. Green indicates above the reference. miRNA, microRNA.

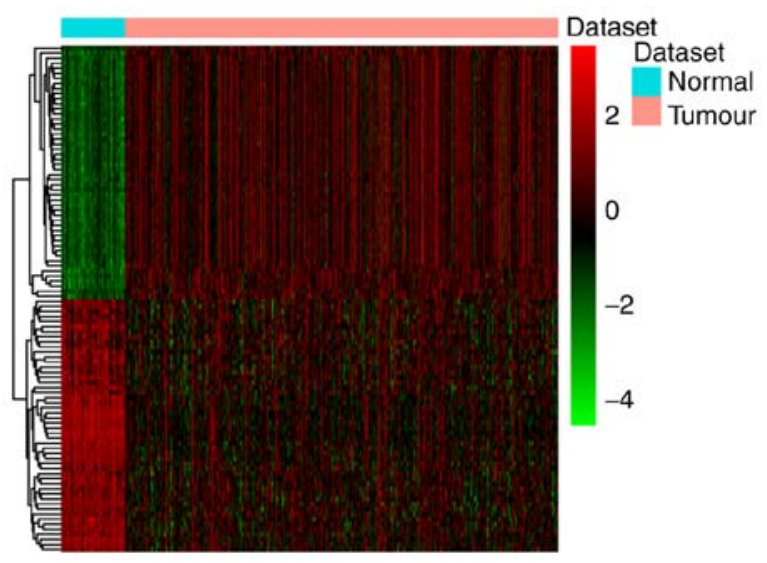

Figure 2. Heat map of the top 100 differentially expressed mRNAs in hepatocellular carcinoma. The diagram shows the result of a two-way hierarchical clustering of the top 100 differentially expressed mRNAs and samples. The clustering was established using the complete-linkage method together with the Euclidean distance. Each row represents a differentially expressed mRNA and each column represents a sample. The differentially expressed mRNA color clustering tree is presented on the right. The color scale illustrates the relative level of differentially expressed mRNA expression. Red indicates below the reference channel. Green indicates above the reference.

and their differentially expressed target mRNAs [secreted frizzled related protein 1 (SFRPI), endothelin receptor type B $(E D N R B)$, nuclear receptor subfamily 4 group A member 3 (NR4A3), four and a half LIM domains 2 (FHL2), NK3 homeobox 1 (NKX3-1), interleukin 6 signal transducer (IL6ST) and forkhead box O1 $(F O X O 1)]$ are presented in Table II. The present results suggested that there was a significantly correlation between the identified miRNAs and mRNAs.

The Starbase database was used to identify the binding sites between the five miRNA and their target mRNAs, and three ATCG base binding sites were found between hsa-miR-183-5p and IL6ST (Fig. 6A), two ATCG base binding sites between hsa-miR-224-5p and NR4A3 (Fig. 6B), two ATCG base 
A

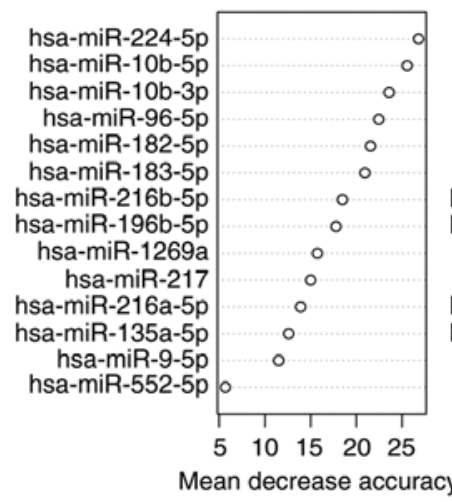

rf_comb

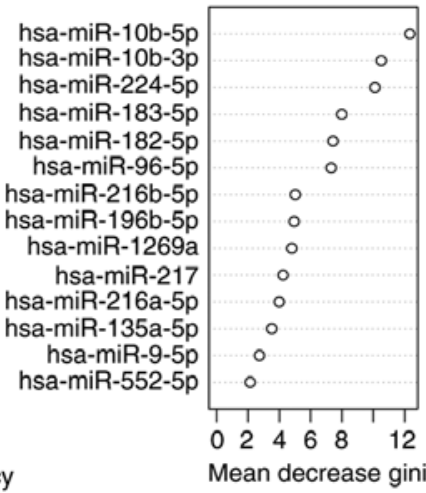

B

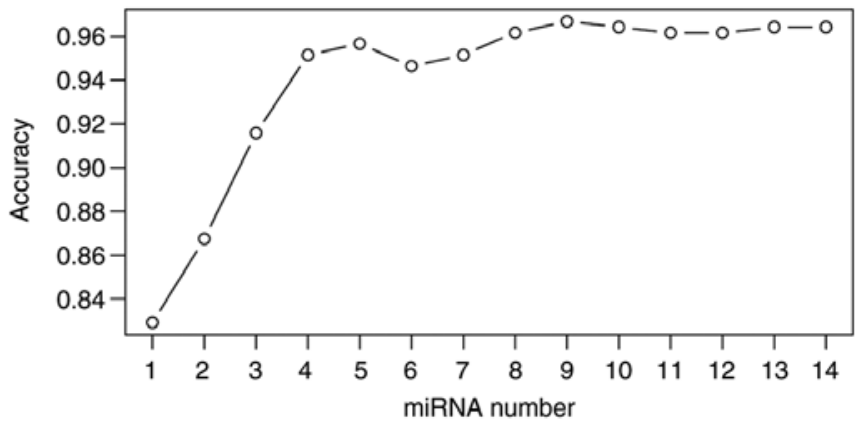

Figure 3. Identification of optimal diagnostic miRNAs biomarkers for hepatocellular carcinoma. (A) Ranking of all differentially expressed miRNAs. Differentially expressed miRNAs were ranked according to the standardized drop in prediction accuracy. (B) Tendency chart of the area under the curve along with the increase of differentially expressed miRNAs. miRNA/miR, microRNA.
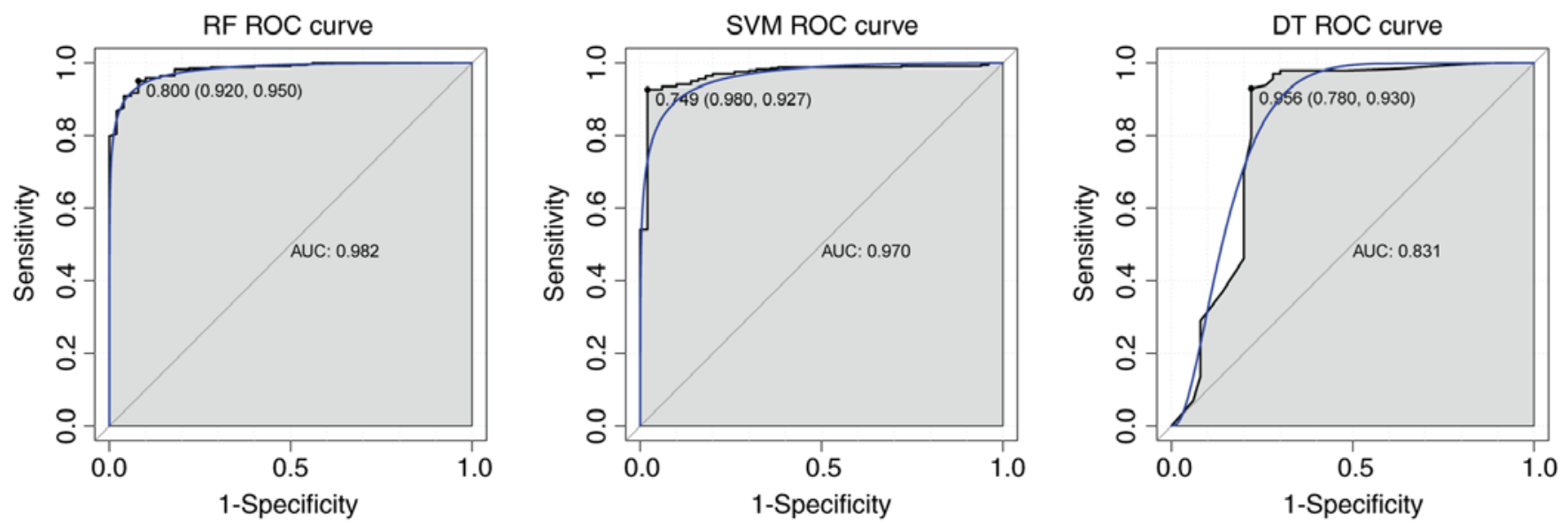

Figure 4. ROC results of the combination of five optimal diagnostic biomarkers, such as hsa-miR-10b-5p, hsa-miR-10b-3p, hsa-miR-182-5p and hsa-miR-183-5p and hsa-miR-224-5p, based on RF, SVM and DT classification models. ROC, receiver operating characteristic; RF, random forests; SVM, support vector machine; DT, decision tree; AUC, area under the curve.

binding sites between hsa-miR-224-5p and FHL2 (Fig. 6C), and two ATCG base binding sites between hsa-miR-182-5p and FOXO1 (Fig. 6D). However, there were no ATCG base binding sites between hsa-miR-224-5p and NKX3-1, hsa-miR-10b-5p and SFRP1, and hsa-miR-10b-3p and EDNRB.

Functional analysis of putative miRNA targets. To understand the potential function of the target differentially expressed mRNAs targeted by hsa-miR-10b-5p, hsa-miR-10b-3p, hsa-miR-224-5p, hsa-miR-183-5p and hsa-miR-182-5p, GO and KEGG pathway analyses were conducted. The present results suggested that these differentially expressed target mRNAs were most significantly enriched in the GO terms of 'cellular response to lipid', 'fat cell differentiation' and 'monocarboxylic acid metabolic process' (Fig. 7A). Additionally, 'bile acid biosynthesis, cholesterol=>cholate/chenodeoxycholate', 'valine, leucine and isoleucine degradation' and 'calcium signaling pathway' were the most enriched signaling pathways according to the KEGG analysis (Fig. 7B).

In silico validation and in vitro validation. The GSE63046 dataset was used to validate the expression levels of
hsa-miR-10b-5p, hsa-miR-10b-3p, hsa-miR-224-5p, hsa-miR-183-5p and hsa-miR-182-5p (Fig. 8). The expression levels of hsa-miR-10b-5p, hsa-miR-10b-3p, hsa-miR-224-5p, hsa-miR-183-5p and hsa-miR-182-5p were all upregulated in tumor tissues compared with normal tissues from the same patients. In addition, in vitro experiments were performed to further validate the expression level of the five differentially expressed miRNAs in seven patients. The clinical information of the seven patients enrolled in the present study is presented in Table III. The expression levels of hsa-miR-10b-5p, hsa-miR-10b-3p, hsa-miR-224-5p, hsa-miR-183-5p and hsa-miR-182-5p were all upregulated (Fig. 9). The validation results were consistent with the present bioinformatics analysis.

In addition, according to the clinical information, patients with hepatocellular carcinoma were divided into two groups: i) Cirrhosis (75 cases); and ii) without cirrhosis (123 cases) to study whether liver cirrhosis may affect the expression of the five differentially expressed miRNAs identified (hsa-miR-10b-5p, hsa-miR-10b-3p, hsa-miR-224-5p, hsa-miR-183-5p and hsa-miR-182-5p) and their target mRNAs (SFRPl, EDNRB, NR4A3, FHL2, NKX3-1, IL6ST and FOXO1). The expression levels of these miRNAs and mRNAs are presented as box 


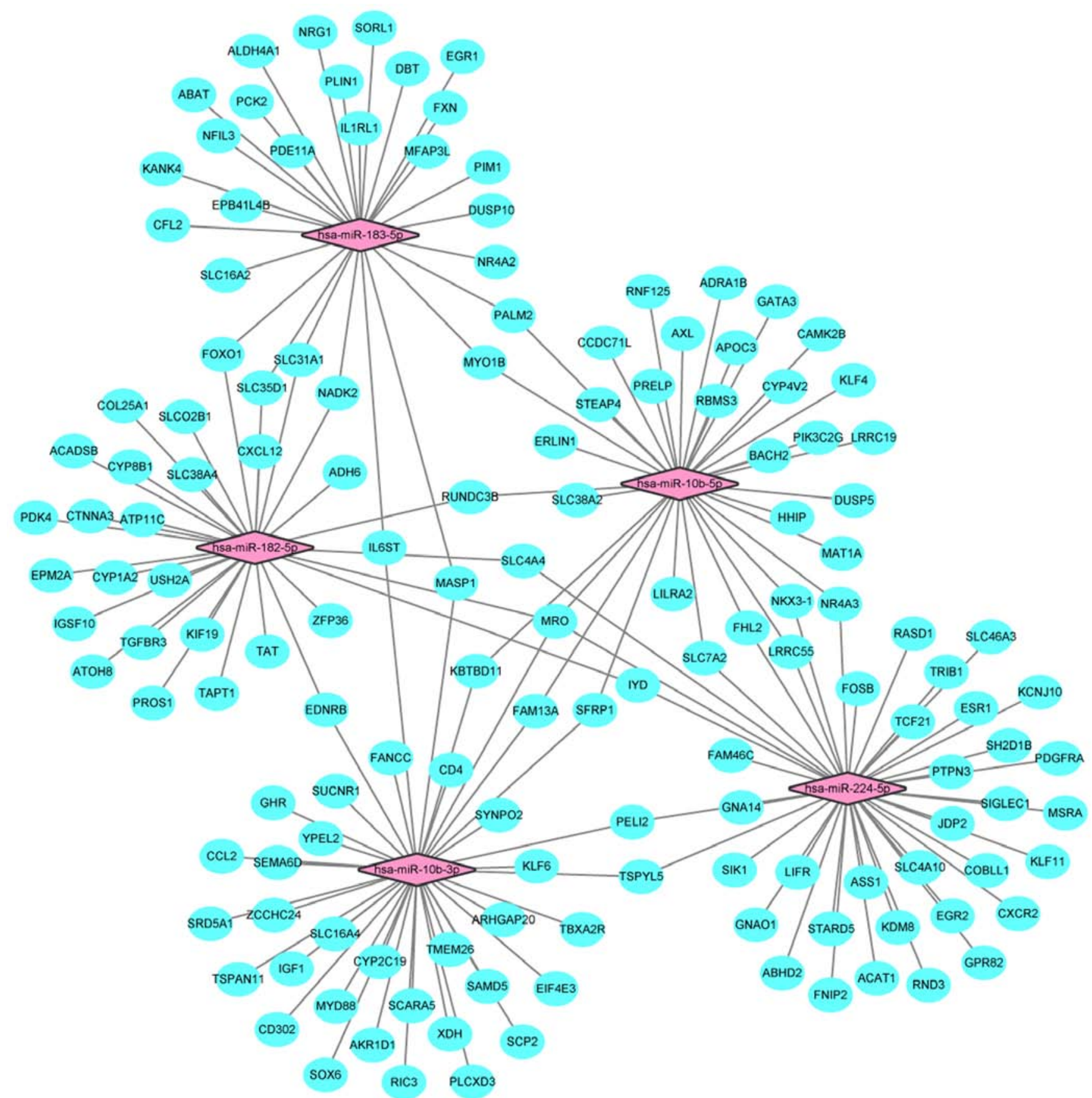

Figure 5. Network of miRNA-target mRNAs with a negative correlation between five differentially expressed miRNAs and 145 differentially expressed miRNAs in hepatocellular carcinoma. The diamonds and ellipses represent the differentially expressed miRNAs and target mRNAs, respectively. Pink and blue represent upregulated miRNA and downregulated mRNA, respectively. miRNA, microRNA.

plots (Fig. 10). The present results suggested that there was no significant difference in these miRNAs and mRNAs, except for FHL2. FHL2 plays a protective mechanistic role during hepatic fibrogenesis (28). Moreover, deficiency in FHL2 aggravates liver fibrosis (28). Collectively, these results suggested that liver cirrhosis may affect special liver cirrhosis-related mRNAs, such as FHL2, without affecting the expression levels of the aforementioned miRNAs and mRNAs identified in patients with hepatocellular carcinoma.

Diagnosis and survival prediction of key differentially expressed miRNAs. ROC curve analysis was performed to assess the diagnosis ability of hsa-miR-10b-5p, hsa-miR-10b-3p, hsa-miR-182-5p, hsa-miR-183-5p and hsa-miR-224-5p (Fig. 11A). The AUC values of hsa-miR-10b-5p (0.889), hsa-miR-10b-3p (0.871) and hsa-miR-224-5p (0.859) were all $>0.8$. For hepatocellular carcinoma diagnosis, the specificity and sensitivity of hsa-miR-10b-5p was 96.0 and $75.7 \%$, respectively; the specificity and sensitivity of hsa-miR-10b-3p was 98.0 and $69.9 \%$, respectively; and the specificity and sensitivity of hsa-miR-224-5p was 96.0 and $70.5 \%$, respectively. In addition, the potential prognostic values of hsa-miR-10b-5p, hsa-miR-10b-3p, hsa-miR-182-5p, hsa-miR-183-5p and hsa-miR-224-5p were analyzed using the online software 

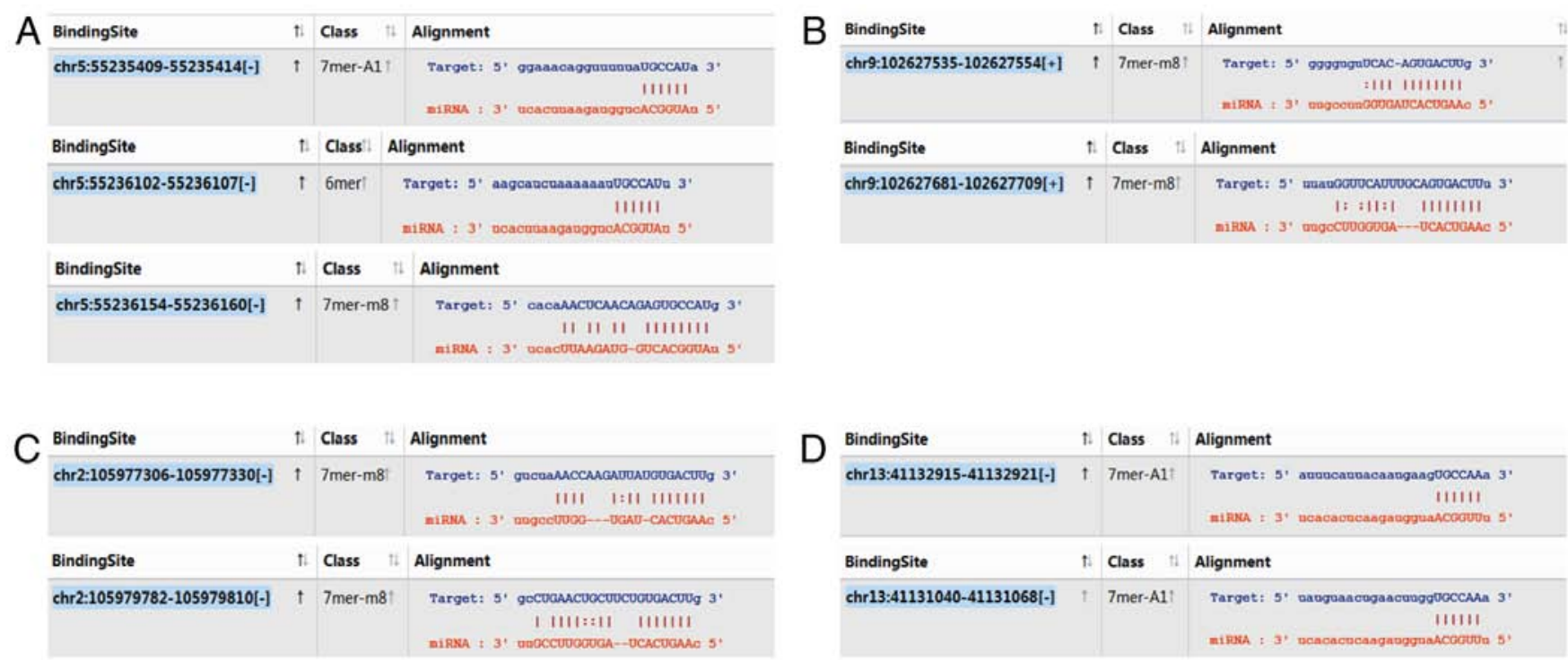

Figure 6. Analysis of the concrete ATCG base binding sites in the miRNA-mRNA pairs identified. (A) ATCG base binding sites between hsa-miR-183-5p and IL6ST. (B) ATCG base binding sites between hsa-miR-224-5p and NR4A3. (C) ATCG base binding sites between hsa-miR-224-5p and FHL2. (D) ATCG base binding sites between hsa-miR-182-5p and FOXO1. miRNA/miR, microRNA; IL6ST, interleukin 6 signal transducer; NR4A3, nuclear receptor subfamily 4 group A member 3; FHL2, four and a half LIM domains 2; FOXO1, forkhead box O1.
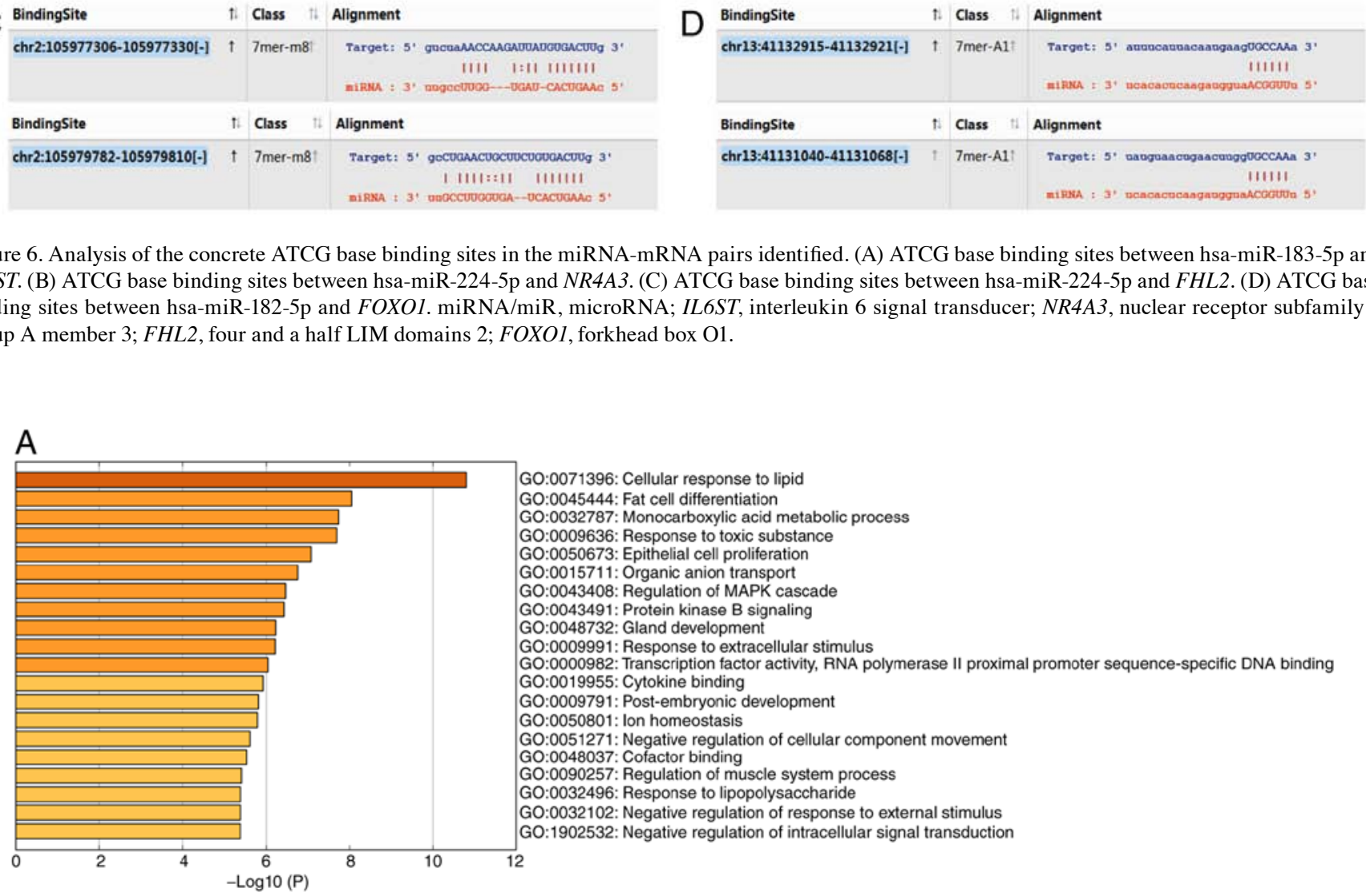

\section{B}

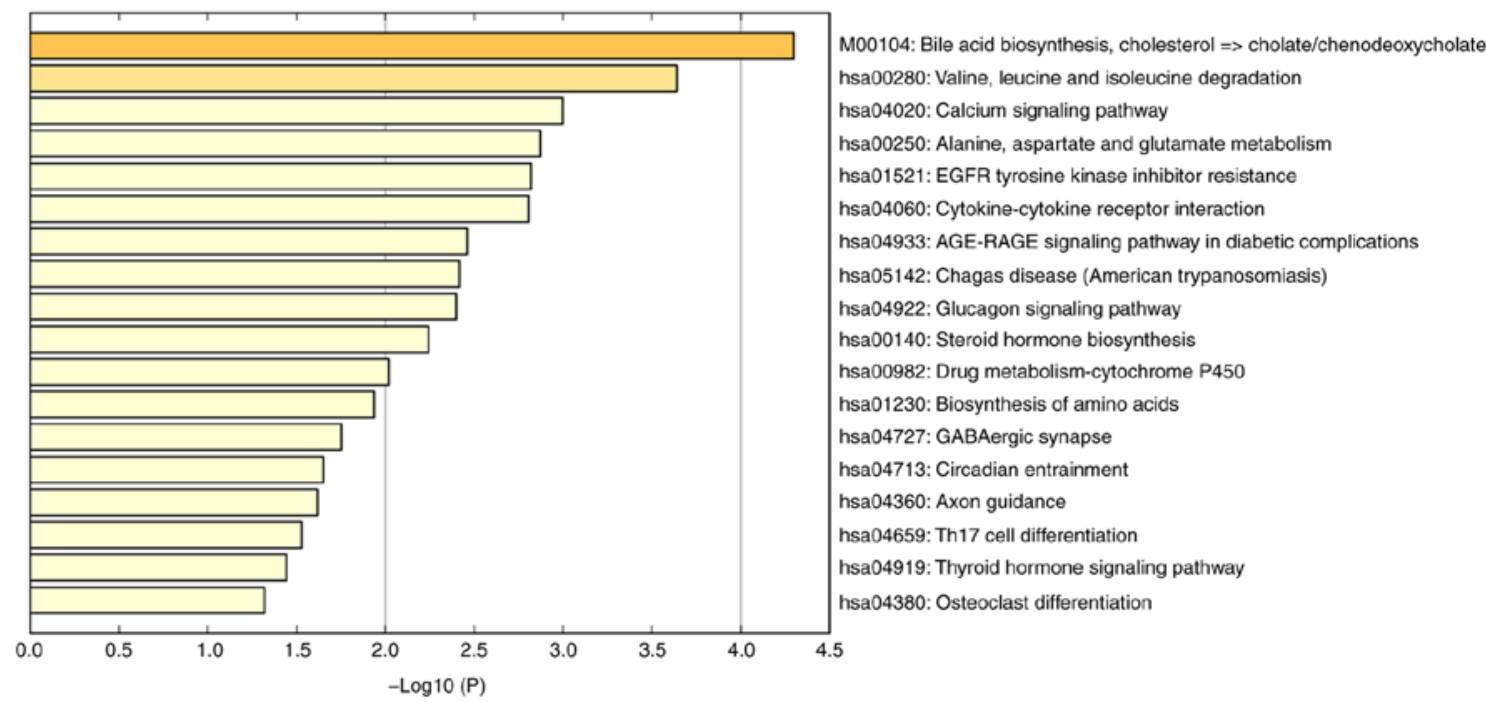

Figure 7. Significantly enriched GO and KEGG terms of differentially expressed mRNAs. (A) Top 20 significantly enriched GO terms of differentially expressed mRNAs. (B) Top 18 significantly enriched KEGG terms of differentially expressed mRNAs. GO, Gene Ontology; KEGG, Kyoto Encyclopedia of Genes and Genomes. 
Table III. Clinical information of seven patients in the reverse transcription-quantitative PCR validation.

\begin{tabular}{|c|c|c|c|c|c|c|c|c|c|}
\hline Patient & Age & Sex & $\begin{array}{l}\text { Family } \\
\text { history }\end{array}$ & $\begin{array}{c}\text { Other } \\
\text { complications }\end{array}$ & $\begin{array}{l}\text { HBV- } \\
\text { infected }\end{array}$ & $\begin{array}{c}\text { HCV- } \\
\text { infected }\end{array}$ & $\begin{array}{c}\text { Alpha } \\
\text { fetoprotein } \\
\text { content, } 400 \mu \mathrm{g} / 1\end{array}$ & $\begin{array}{l}\text { Imaging } \\
\text { examination } \\
\text { results }\end{array}$ & Therapy history \\
\hline 1 & 55 & Male & No & Liver cirrhosis & Yes & No & 387.38 & $\begin{array}{l}\text { Primary } \\
\text { hepatocellular } \\
\text { carcinoma }\end{array}$ & $\begin{array}{l}\text { Liver } \\
\text { transplantation }\end{array}$ \\
\hline 2 & 71 & Male & No & Liver cirrhosis & No & No & 4.83 & $\begin{array}{l}\text { Primary } \\
\text { hepatocellular } \\
\text { carcinoma }\end{array}$ & Hepatectomy \\
\hline 3 & 74 & Male & No & Liver cirrhosis & No & No & 1.24 & $\begin{array}{l}\text { Primary } \\
\text { hepatocellular } \\
\text { carcinoma }\end{array}$ & Hepatectomy \\
\hline 4 & 56 & Male & No & Liver cirrhosis & No & No & 39.28 & $\begin{array}{l}\text { Primary } \\
\text { hepatocellular } \\
\text { carcinoma }\end{array}$ & $\begin{array}{l}\text { Liver } \\
\text { transplantation }\end{array}$ \\
\hline 5 & 65 & Male & No & Liver cirrhosis & No & No & 2.48 & $\begin{array}{l}\text { Primary } \\
\text { hepatocellular } \\
\text { carcinoma }\end{array}$ & Hepatectomy \\
\hline 6 & 50 & Male & No & Liver cirrhosis & Yes & No & 107.08 & $\begin{array}{l}\text { Primary } \\
\text { hepatocellular } \\
\text { carcinoma }\end{array}$ & Hepatectomy \\
\hline 7 & 54 & Male & No & Liver cirrhosis & Yes & No & 1.48 & $\begin{array}{l}\text { Primary } \\
\text { hepatocellular } \\
\text { carcinoma }\end{array}$ & $\begin{array}{l}\text { Liver } \\
\text { transplantation }\end{array}$ \\
\hline
\end{tabular}

HBV, hepatitis B virus; HCV, hepatitis C virus.
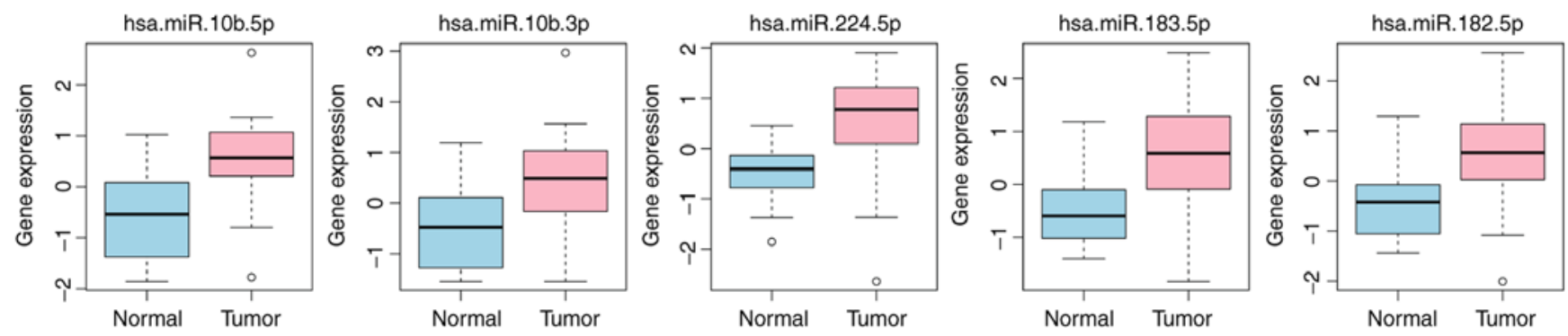

Figure 8. Expression box plots of hsa-miR-10b-5p, hsa-miR-10b-3p, hsa-miR-224-5p, hsa-miR-183-5p and hsa-miR-182-5p in the GSE63046 dataset. miR, microRNA.

survival package in $\mathrm{R}$ (https://cran.r-project.org/web/packages/survival/index.html). The present results demonstrated that hsa-miR-10b-5p and hsa-miR-10b-3p were considered to be significantly negatively associated with survival $(\mathrm{P}<0.05)$ in patients with hepatocellular carcinoma. The survival curves of hsa-miR-10b-5p, hsa-miR-10b-3p, hsa-miR-182-5p, hsa-miR-183-5p and hsa-miR-224-5p are presented in Fig. 11B.

\section{Discussion}

In the present study, hsa-miR-10b-5p, hsa-miR-10b-3p, hsa-miR-224-5p, hsa-miR-183-5p and hsa-miR-182-5p were considered as optimal diagnostic biomarkers for hepatocellular carcinoma based on machine learning approaches. hsa-miR-10b-5p was upregulated in advanced liver fibrosis (29). In addition, higher levels of expression of hsa-miR-10b-5p were identified in hepatocellular carcinoma cell lines (30). A previous study suggested that hsa-miR-10b-5p may be associated with the invasion and migration in hepatocellular carcinoma (31). In the present study, it was additionally identified that hsa-miR-10b-5p upregulated in hepatocellular carcinoma, which was consistent with a previous study (30). Notably, it was identified that hsa-miR-10b-5p exhibited a diagnostic and prognostic value in patients with hepatocellular carcinoma. In addition, SFRPI was one of the target mRNAs of hsa-miR-10b-5p. SFRP1, a putative tumor suppressor protein, 


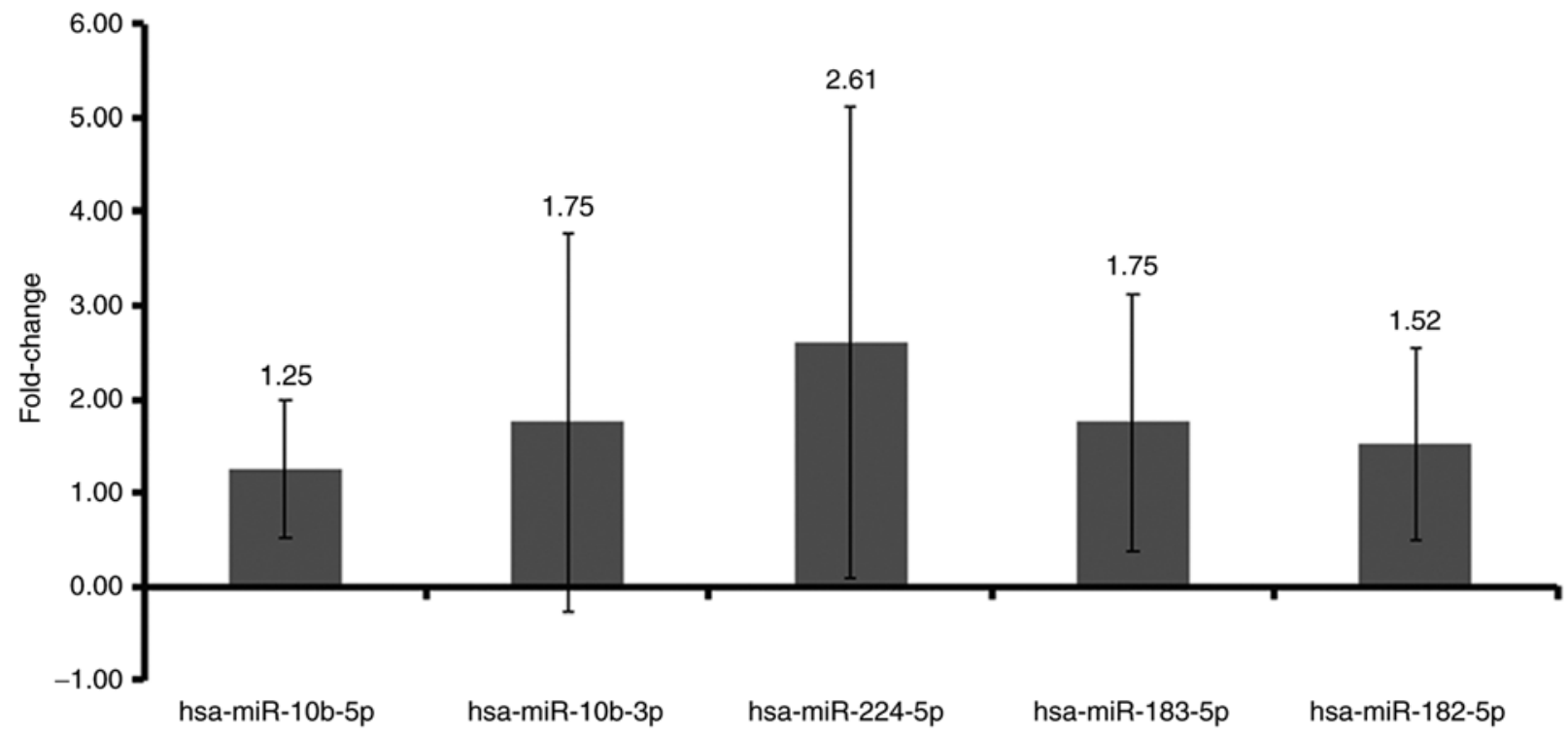

Figure 9. In vitro reverse transcription-quantitative PCR validation of hsa-miR-10b-5p, hsa-miR-10b-3p, hsa-miR-224-5p, hsa-miR-183-5p and hsa-miR-182-5p in tumor samples. Fold change $>1$ and fold change $<1$ represents upregulation and downregulation, respectively. miR, microRNA.

\section{A}
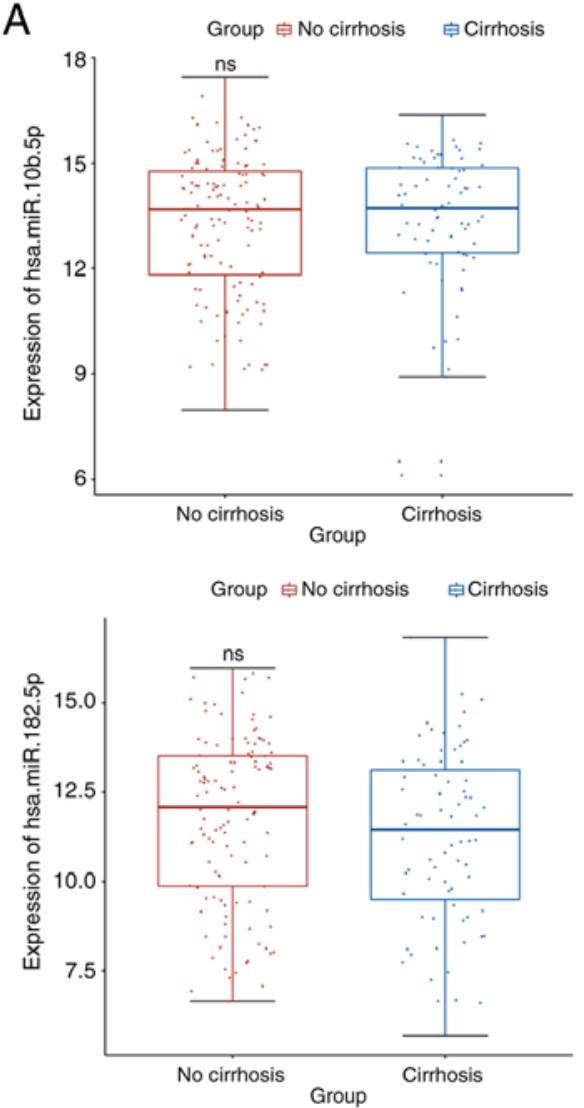
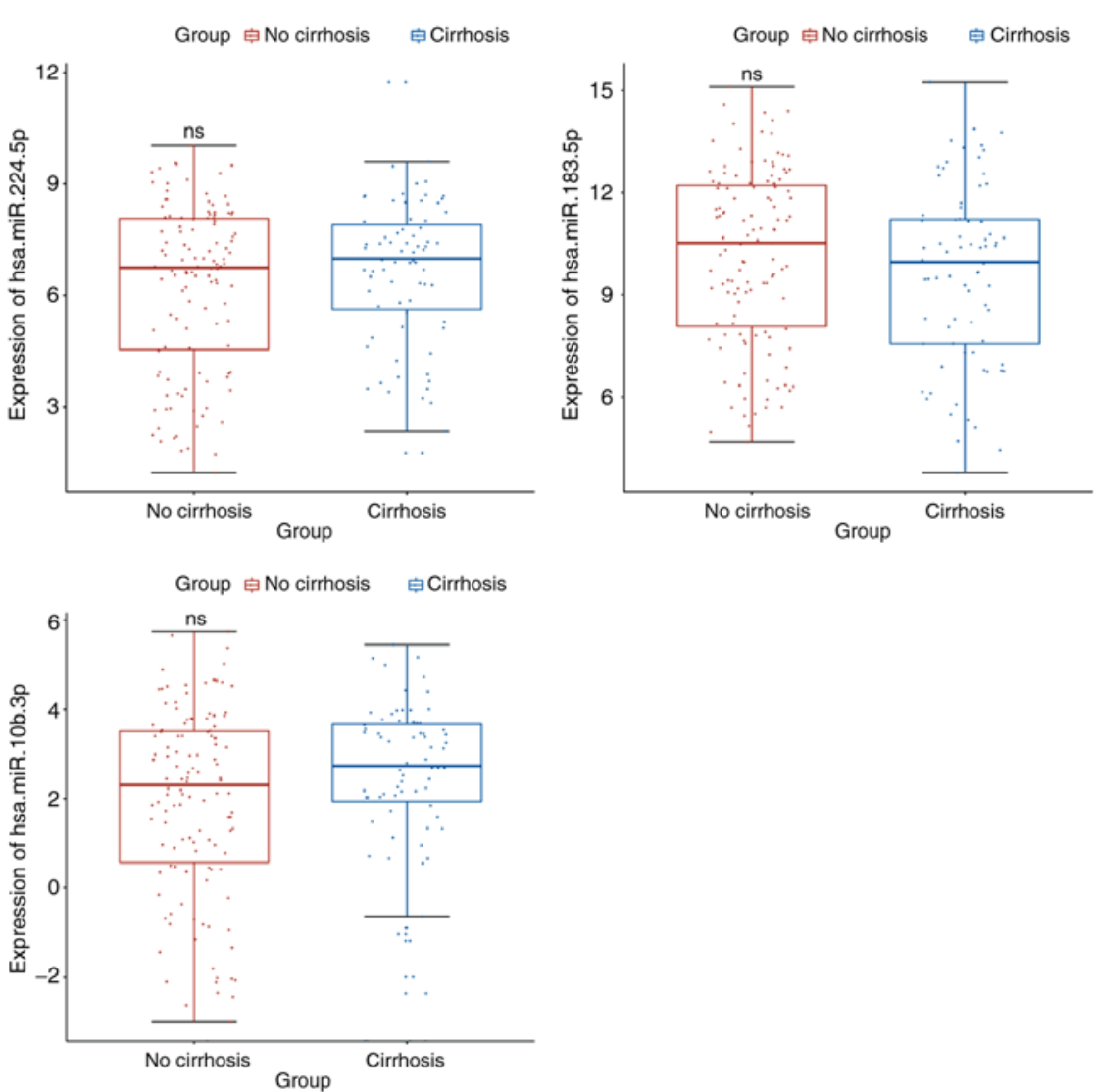

Figure 10. Box plots of selected miRNAs and mRNAs. (A) Box plots of hsa-miR-10b-5p, hsa-miR-10b-3p, hsa-miR-224-5p, hsa-miR-183-5p and hsa-miR-182-5p in liver cirrhosis.

is decreased in hepatocellular carcinoma (32). A previous study suggested that SFRP1 expression may be downregulated by methylation levels, which may activate the Wnt signaling pathway, and increase cell growth and proliferation in hepatocellular carcinoma (33). Huang et al (34) identified that SFRP1 was a potential diagnostic biomarker for hepatocellular carcinoma. The present results suggested that hsa-miR-10b-5p may regulate cell growth and proliferation of hepatocellular carcinoma by targeting SFRP1.

A previous study demonstrated that hsa-miR-10b-3p plays an important role in tumor growth and metastasis (35). hsa-miR-10b-3p was upregulated in 

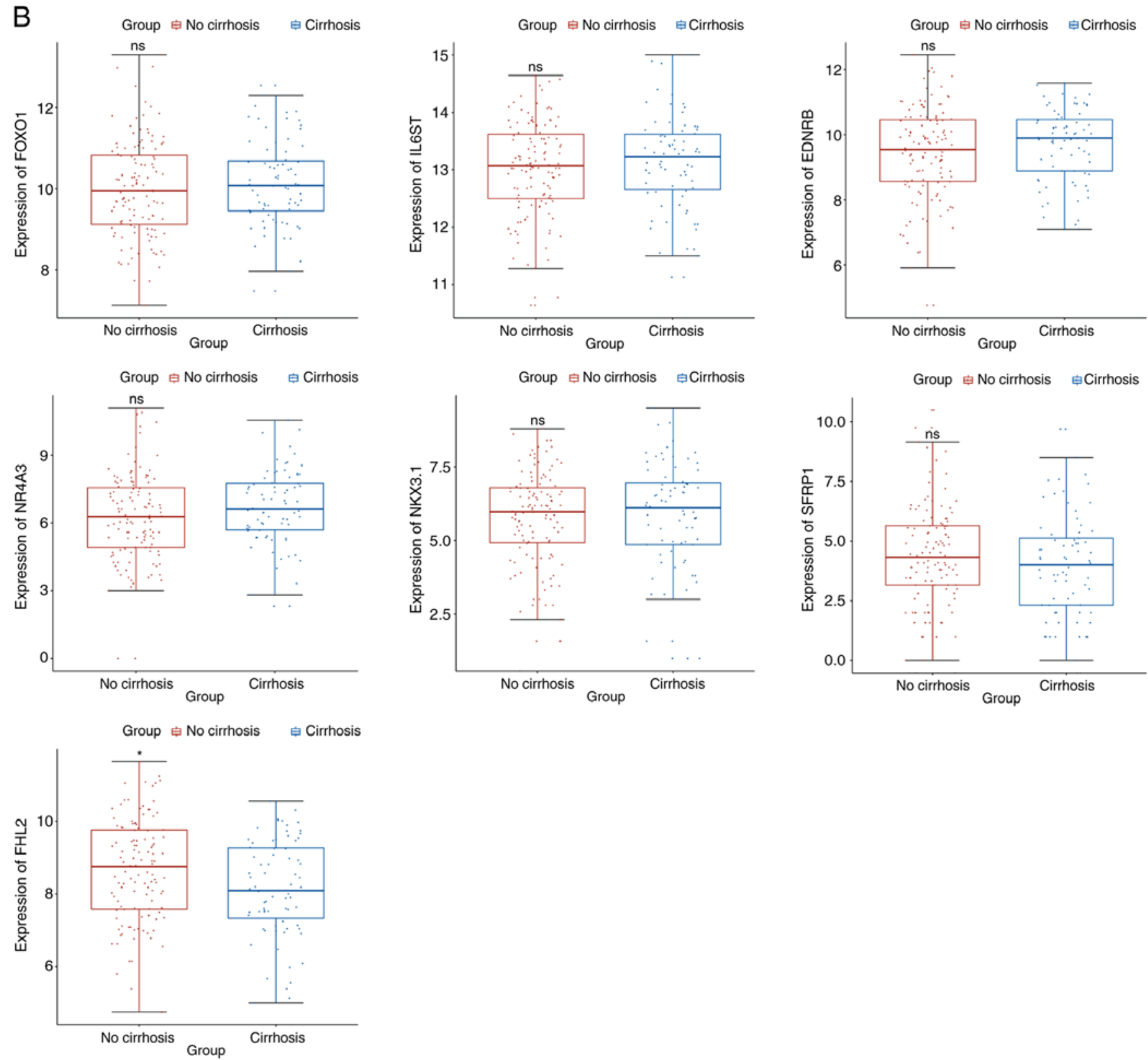

Figure 10. Continued. Box plots of selected miRNAs and mRNAs. (B) Box plots of SFRP1, EDNRB, NR4A3, FHL2, NKX3-1, IL6ST and FOXO1 in liver cirrhosis. " $\mathrm{P}<0.05$ vs. cirrhosis. miR, microRNA; SFRPI, secreted frizzled related protein 1 ; EDNRB, endothelin receptor type B; NR4A3, nuclear receptor subfamily 4 group A member 3; FHL2, four and a half LIM domains 2; NKX3-1, NK3 homeobox 1; IL6ST, interleukin 6 signal transducer; FOXO1, forkhead box O1; ns, not significant.

hepatocellular carcinoma (36). Moreover, the upregulation of hsa-miR-10b-3p was associated with the diagnosis and prognosis of hepatocellular carcinoma $(36,37)$. Similarly, the present study identified that hsa-miR-10b-3p was upregulated in hepatocellular carcinoma. Furthermore, hsa-miR-10b-3p had a significant diagnostic and prognostic value for patients with hepatocellular carcinoma. The present results suggested the important role of hsa-miR-10b-3p in the development of hepatocellular carcinoma. In the target analysis, $E D N R B$ was identified as a target mRNA of hsa-miR-10b-3p. EDNRB, a tumor suppressor, is very frequently methylated in hepatocellular carcinoma tissues (14). EDNRB was previously identified as a biomarker for hepatocellular carcinoma (38). This suggested that hsa-miR-10b-3p may serve a crucial role in the process of hepatocellular carcinoma by regulating EDNRB.

hsa-miR-224-5p wass upregulated in both tumor tissues and blood in patients with hepatocellular carcinoma (39-41). In addition, hsa-miR-224-5p was significantly associated with survival rate in patients with hepatocellular carcinoma (42). Similarly, the present study identified that hsa-miR-224-5p was upregulated in hepatocellular carcinoma tumor. In addition, $N R 4 A 3, F H L 2$ and $N K X 3-1$ were three of the target mRNAs of hsa-miR-224-5p. NR4A3, a transcription factor, is a regulator of hepatoma cell and is associated with survival time in patients with hepatocellular carcinoma $(43,44)$. FHL2 is an anti-proliferative- and metastasis-associated gene $(45,46)$. It was identified that FHL2 plays a protective mechanistic role during hepatic 
A

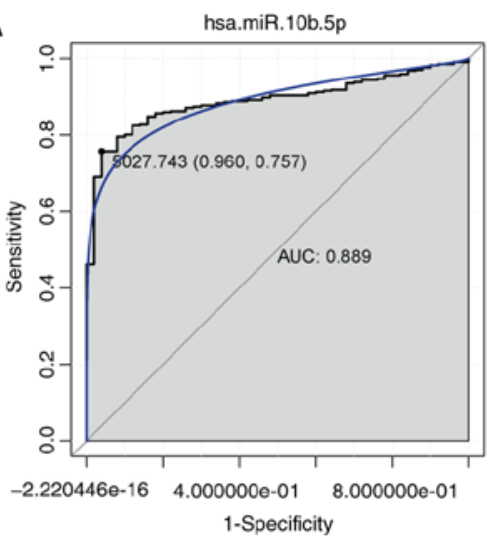

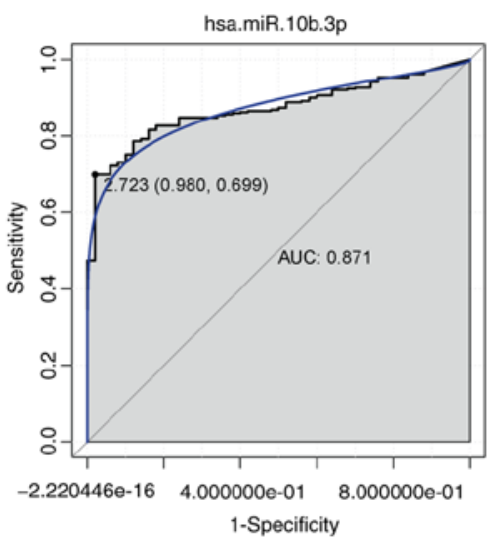
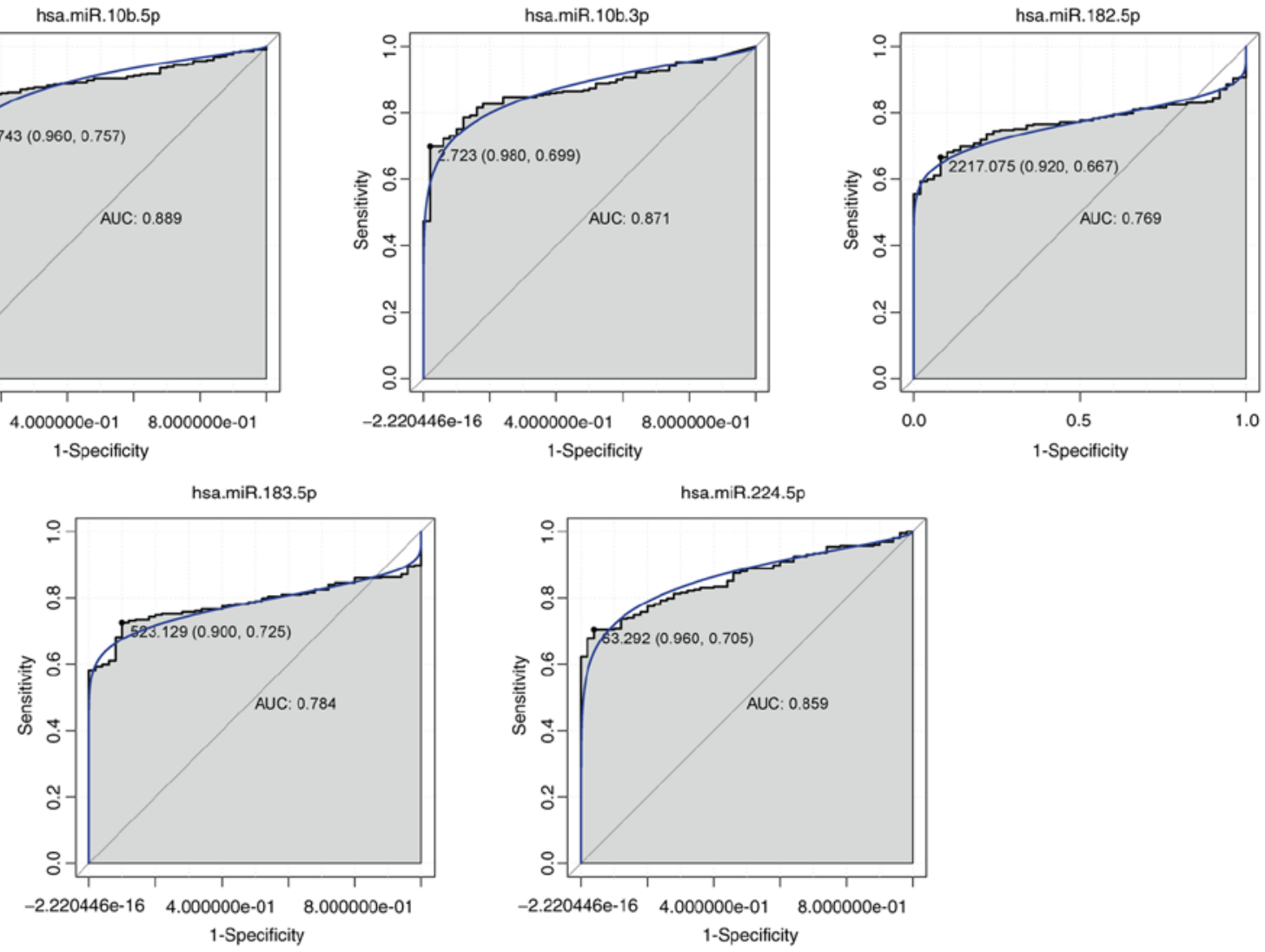

B
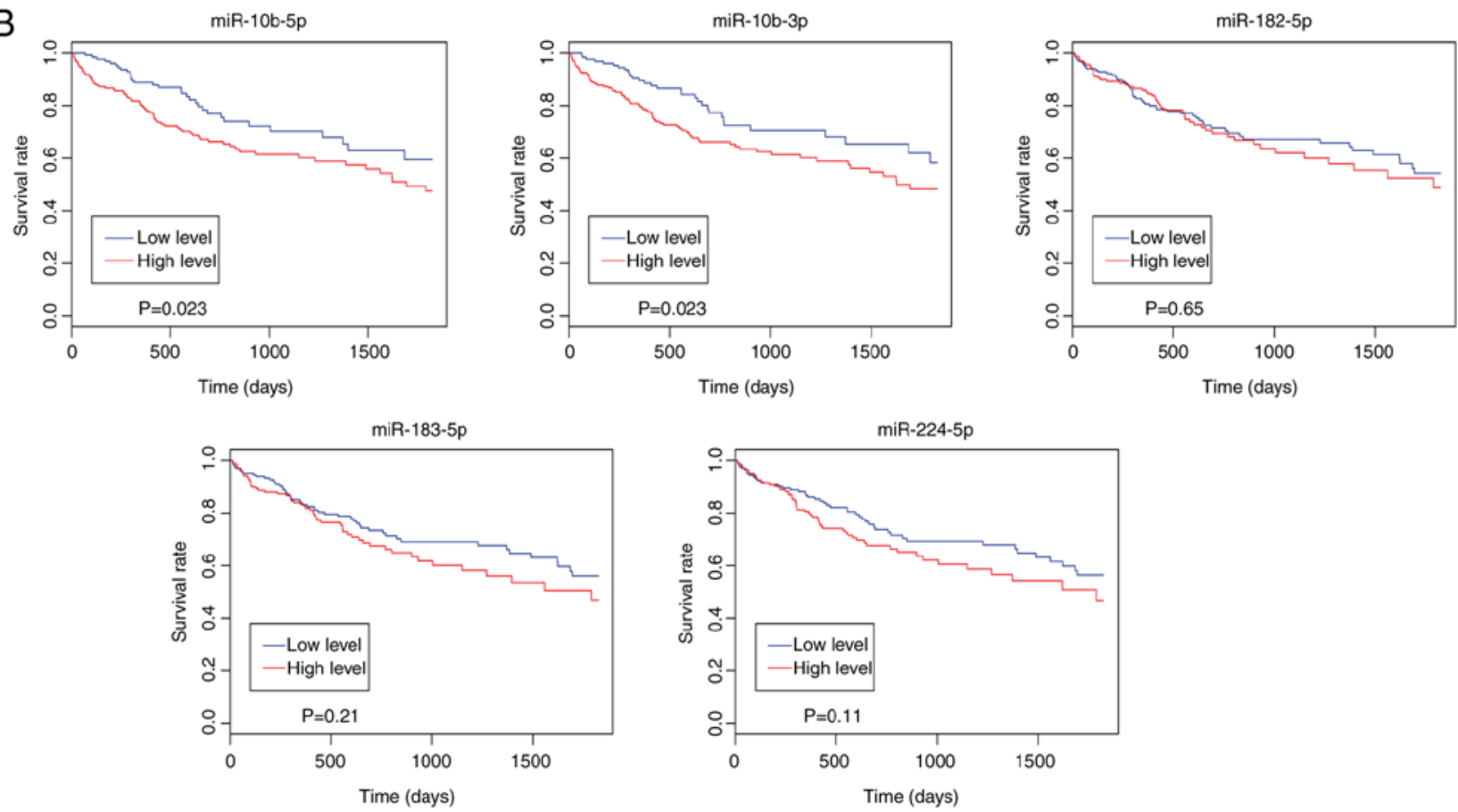

Figure 11. Diagnostic and survival analysis of hsa-miR-10b-5p, hsa-miR-10b-3p, hsa-miR-182-5p, hsa-miR-183-5p and hsa-miR-224-5p. (A) ROC curves of hsa-miR-10b-5p, hsa-miR-10b-3p, hsa-miR-182-5p, hsa-miR-183-5p and hsa-miR-224-5p between patients with hepatocellular carcinoma and healthy controls. ROC curves were used to show the diagnostic ability with 1-specificity and sensitivity. (B) Survival curves of hsa-miR-10b-5p, hsa-miR-10b-3p, hsa-miR-182-5p, hsa-miR-183-5p and hsa-miR-224-5p in patients with hepatocellular carcinoma. Survival curves were used to show the survival ability with time and survival rate. ROC, receiver operating characteristic; miR, microRNA; AUC, area under the curve.

fibrogenesis (28). Moreover, deficiency in FHL2 aggravates liver fibrosis (28). The expression level of FHL2 is downregulated in most patients with hepatocellular carcinoma (47). NKX3-1, a tumor suppressor, is associated with liver fibrosis (48). Aberrant methylation of NKX3-1 was observed in hepatocellular carcinoma (49). It was previously suggested that $N K X 3-1$ is a potential predictor of patients with hepatocellular carcinoma recurrence (50). Investigating 
the regulation between hsa-miR-224-5p and NR4A3, FHL2 and $N K X 3-1$ may provide insight for the understanding of the molecular mechanism underlying hepatocellular carcinoma.

hsa-miR-183-5p was upregulated in advanced liver fibrosis and hepatocellular carcinoma tissue $(26,51)$. Leung et al (52) identified that high expression of hsa-miR-183-5p was significantly associated with invasion and metastasis, and may be a potential biomarker for the survival time of patients with hepatocellular carcinoma. In the present study, it was demonstrated that hsa-miR-183-5p was upregulated in hepatocellular carcinoma, in agreement with the aforementioned previous studies. Moreover, IL6ST was one of the target mRNAs of hsa-miR-183-5p. IL6ST is a gene involved in liver development (53). Changes in IL6ST were significantly associated with hepatotoxicity, including liver damage, inflammation and fibrosis (54). Alterations in IL6ST were frequently observed in hepatocellular adenoma (55). In addition, frequent upregulation and mutations in IL6ST were also detected in hepatocellular carcinoma (56). The present study suggested that hsa-miR-183-5p served a crucial role in the development of hepatocellular carcinoma by targeting IL6ST.

hsa-miR-182-5p was upregulated in hepatocellular carcinoma tissues and cell lines $(51,57)$. hsa-miR-182-5p was associated with hepatocellular carcinoma metastasis, and could be a potential diagnostic and prognostic biomarker in patients with hepatocellular carcinoma $(57,58)$. In addition, hsa-miR-182-5p may be a predictor of early recurrence in patients with hepatocellular carcinoma undergoing surgery (57). In the present study, the expression of hsa-miR-182-5p was increased in hepatocellular carcinoma. Additionally, FOXOI was predicted to be regulated by hsa-miR-182-5p. FOXO1 is one of the most abundantly expressed genes in the liver, and regulates the expression of genes involved in cell cycle, metabolism and differentiation (59). Calvisi et al (60) demonstrated that the expression level of $\mathrm{FOXO1}$ was downregulated in hepatocellular carcinoma. FOXO1 could reverse epithelial-interstitial transformation by inhibiting invasion and metastasis in hepatocellular carcinoma cells (61). FOXO1 was previously considered as a prognostic biomarker and potential target for hepatocellular carcinoma (62). The present findings suggested that the assocation between hsa-miR-182-5p and FOXO1 was associated with hepatocellular carcinoma.

According to the KEGG pathway analysis performed on the mRNAs targeted by the five key miRNAs, "bile acid biosynthesis, cholesterol' was the most enriched signaling pathway. Bile acids are essential for protecting the liver from cholesterol. Bile acid metabolism is significantly regulated by enzymes involved in the liver. Bile acids could be highly toxic if accumulated in high concentrations in the liver. Additionally, a previous study demonstrated that bile acids are promoters of hepatocarcinogenesis (63-65). In addition, cholesterol intake is an independent risk factor for hepatocellular carcinoma (66-68).

Collectively, a number of differentially expressed miRNAs and mRNAs were identified in the present study. According to a machine learning approach, hsa-miR-10b-5p, hsa-miR-10b-3p, hsa-miR-224-5p, hsa-miR-183-5p and hsa-miR-182-5p were considered as optimal diagnostic biomarkers for hepatocellular carcinoma. The genes targeted and downregulated by these five miRNAs, including SFRP1, EDNRB, NR4A3, FHL2, NKX3-1, IL6ST and FOXO1, may be involved in hepatocellular carcinoma tumorigenesis. However, there are certain limitations in the present study. The sample size in the RT-qPCR experiments was small and larger numbers of tumor tissues are required for validating the data of the present study. The molecular mechanisms of the differentially expressed miRNAs and mRNAs identified in hepatocellular carcinoma tumorigenesis were not investigated. Additional in vitro experiments, such as cell culture and establishment of an animal model, are required to further investigate the potential mechanisms underlying the disease. Furthermore, the minimally invasive diagnostic methods for hepatocellular carcinoma were lacking and the potential use of miRNAs as blood/serum markers of hepatocellular carcinoma requires further examination. The present study may provide research basis for the diagnosis and treatment of hepatocellular carcinoma.

\section{Acknowledgements}

Not applicable.

\section{Funding}

The present study was funded by Provincial Outstanding Clinical Medicine Talents (Medical Leading Talents).

\section{Availability of data and materials}

The datasets used and/or analyzed during the current study are available from the corresponding author on reasonable request.

\section{Authors' contributions}

XZ, JD, JC, YW, QG, QZ, WL, BL, ZC, LT and JZ analyzed and interpreted the data. $\mathrm{XZ}$ and $\mathrm{CZ}$ wrote, edited and revised the manuscript. $\mathrm{CZ}$ proposed the conception fo the study and designed the project. All authors read and approved the final manuscript.

\section{Ethics approval and consent to participate}

The present study was approved by The Institutional Ethics Review Board of The Third Hospital of Hebei Medical University (approval no. 2018-025-1). In addition, informed consent was obtained from the individuals.

\section{Patient consent for publication}

Informed written consent was obtained from all subjects.

\section{Competing interests}

The authors declare that they have no competing interests.

\section{References}

1. Torre LA, Bray F, Siegel RL, Ferlay J, Lortet-Tieulent J and Jemal A: Global cancer statistics, 2012. CA Cancer J Clin 65: 87-108, 2015. 
2. Critelli RM, De Maria N and Villa E: Biology of hepatocellular carcinoma. Dig Dis 33: 635-641, 2015.

3. Forner A, Llovet JM and Bruix J: Hepatocellular carcinoma. Lancet 379: 1245-1255, 2012.

4. Fattovich G, Stroffolini T, Zagni I and Donato F: Hepatocellular carcinoma in cirrhosis: Incidence and risk factors. Gastroenterology 127(5 Suppl 1): S35-S50, 2004.

5. Kumagi T, Hiasa Y and Hirschfield GM: Hepatocellular carcinoma for the non-specialist. BMJ 339: b5039, 2009.

6. Tanaka M, Katayama F, Kato H, Tanaka H, Wang J, Qiao YL and Inoue M: Hepatitis B and $\mathrm{C}$ virus infection and hepatocellular carcinoma in China: A review of epidemiology and control measures. J Epidemiol 21: 401-416, 2011.

7. El-Serag HB and Rudolph KL: Hepatocellular carcinoma: Epidemiology and molecular carcinogenesis. Gastroenterology 132: 2557-2576, 2007.

8. Llovet JM, Zucman-Rossi J, Pikarsky E, Sangro B, Schwartz M, Sherman M and Gores G: Hepatocellular carcinoma. Nat Rev Dis Primer 2: 16018, 2016.

9. Nishida N, Nagasaka T, Nishimura T, Ikai I, Boland CR and Goel A: Aberrant methylation of multiple tumor suppressor genes in aging liver, chronic hepatitis, and hepatocellular carcinoma. Hepatology 47: 908-918, 2008.

10. Lehmann U, Wingen LU, Brakensiek K, Wedemeyer $\mathrm{H}$ Becker T, Heim A, Metzig K, Hasemeier B, Kreipe H and Flemming P: Epigenetic defects of hepatocellular carcinoma are already found in non-neoplastic liver cells from patients with hereditary haemochromatosis. Hum Mol Genet 16 : 1335-1342, 2007.

11. Li B, Liu W, Wang L, Li M, Wang J, Huang L, Huang P and Yuan Y: CpG island methylator phenotype associated with tumor recurrence in tumor-node-metastasis stage I hepatocellular carcinoma. Ann Surg Oncol 17: 1917-1926, 2010.

12. Liu H, Wang X, Feng B, Tang L, Li W, Zheng X, Liu Y, Peng Y, Zheng $\mathrm{G}$ and $\mathrm{He}$ Q: Golgi phosphoprotein 3 (GOLPH3) promotes hepatocellular carcinoma progression by activating mTOR signaling pathway. BMC Cancer 18: 661, 2018.

13. Roxburgh P and Evans TR: Systemic therapy of hepatocellular carcinoma: Are we making progress? Adv Ther 25: 1089-1104, 2008.

14. Chen H, Zhang T, Sheng Y, Zhang C, Peng Y, Wang X and Zhang C: Methylation profiling of multiple tumor suppressor genes in hepatocellular carcinoma and the epigenetic mechanism of 3OST2 regulation. J Cancer 6: 740-749, 2015.

15. Plasterk RH: Micro RNAs in animal development. Cell 124 877-881, 2006.

16. Tang W, Tang J, He J, Zhou Z, Qin Y, Qin J, Li B, Xu X, Geng Q, Jiang W, et al: SLIT2/ROBO1-miR-218-1-RET/PLAG1: A new disease pathway involved in hirschsprung's disease. J Cell Mol Med 19: 1197-1207, 2015.

17. Xu J, Wu C, Che X, Wang L, Yu D, Zhang T, Huang L, Li H, Tan W, Wang C and Lin D: Circulating microRNAs, miR-21, miR-122, and miR-223, in patients with hepatocellular carcinoma or chronic hepatitis. Mol Carcinog 50: 136-142, 2011.

18. Tomimaru Y, Eguchi $\mathrm{H}$, Nagano $\mathrm{H}$, Wada H, Kobayashi $\mathrm{S}$ Marubashi S, Tanemura M, Tomokuni A, Takemasa I, Umeshita K, et al: Circulating microRNA-21 as a novel biomarker for hepatocellular carcinoma. J Hepatol 56: 167-175, 2012.

19. Zhang J, Yang Y, Yang T, Liu Y, Li A, Fu S, Wu M, Pan Z and Zhou W: MicroRNA-22, downregulated in hepatocellular carcinoma and correlated with prognosis, suppresses cell proliferation and tumourigenicity. Br J Cancer 103: 1215-1220, 2010.

20. Huang J, Wang Y, Guo Y and Sun S: Down-regulated microRNA-152 induces aberrant DNA methylation in hepatitis $B$ virus-related hepatocellular carcinoma by targeting DNA methyltransferase 1. Hepatology 52: 60-70, 2010

21. Gramantieri L, Ferracin M, Fornari F, Veronese A, Sabbioni S, Liu CG, Calin GA, Giovannini C, Ferrazzi E, Grazi GL, et al: Cyclin G1 is a target of miR-122a, a microRNA frequently down-regulated in human hepatocellular carcinoma. Cancer Res 67: 6092-6099, 2007.

22. Callegari E, Gramantieri L, Domenicali M, D'Abundo L, Sabbioni S and Negrini M: MicroRNAs in liver cancer: A model for investigating pathogenesis and novel therapeutic approaches. Cell Death Differ 22: 46-57, 2015

23. Anders $\mathrm{S}$ and Huber W: Differential expression analysis for sequence count data. Genome Biol 11: R106, 2010.

24. Benjamini Y and Hochberg Y: Controlling the false discovery rate: A practical and powerful approach to multiple testing. J $\mathrm{R}$ Stat Soc B 57: 289-300, 1995.
25. Smoot ME, Ono K, Ruscheinski J, Wang PL and Ideker T: Cytoscape 2.8: New features for data integration and network visualization. Bioinformatics 27: 431-432, 2011.

26. Wojcicka A, Swierniak M, Kornasiewicz O, Gierlikowski W, Maciag M, Kolanowska M, Kotlarek M, Gornicka B, Koperski L, Niewinski $\mathrm{G}$, et al: Next generation sequencing reveals microRNA isoforms in liver cirrhosis and hepatocellular carcinoma. Int J Biochem Cell Biol 53: 208-217, 2014.

27. Livak KJ and Schmittgen TD: Analysis of relative gene expression data using real-time quantitative PCR and the 2(-Delta Delta C(T)) method. Methods 25: 402-408, 2001

28. Huss S, Stellmacher C, Goltz D, Khlistunova I, Adam AC, Trebicka J, Kirfel J, Büttner R and Weiskirchen R: Deficiency in four and one half LIM domain protein 2 (FHL2) aggravates liver fibrosis in mice. BMC Gastroenterol 13: 8, 2013.

29. Coppola N, Onorato L, Panella M, de Stefano G, Mosca N, Minichini C, Messina V, Potenza N, Starace M, Alessio L, et al: Correlation between the hepatic expression of human MicroRNA hsa-miR-125a-5p and the progression of fibrosis in patients with overt and occult HBV infection. Front Immunol 9: 1334,2018

30. Wei JX, Lv LH, Wan YL, Cao Y, Li GL, Lin HM, Zhou R, Shang CZ, Cao J, He H, et al: Vps4A functions as a tumor suppressor by regulating the secretion and uptake of exosomal microRNAs in human hepatoma cells. Hepatology 61: 1284-1294, 2015.

31. Li QJ, Zhou L, Yang F, Wang GX, Zheng H, Wang DS, He Y and Dou KF: MicroRNA-10b promotes migration and invasion through CADM1 in human hepatocellular carcinoma cells. Tumour Biol 33: 1455-1465, 2012.

32. Finch PW, He X, Kelley MJ, Uren A, Schaudies RP, Popescu NC, Rudikoff S, Aaronson SA, Varmus HE and Rubin JS: Purification and molecular cloning of a secreted, Frizzled-related antagonist of Wnt action. Proc Natl Acad Sci USA 94: 6770-6775, 1997.

33. Kaur P, Mani S, Cros MP, Scoazec JY, Chemin I, Hainaut P and Herceg Z: Epigenetic silencing of sFRP1 activates the canonical Wnt pathway and contributes to increased cell growth and proliferation in hepatocellular carcinoma. Tumour Biol 33: 325-336, 2012.

34. Huang ZH, Hu Y, Hua D, Wu YY, Song MX and Cheng ZH: Quantitative analysis of multiple methylated genes in plasma for the diagnosis and prognosis of hepatocellular carcinoma. Exp Mol Pathol 91: 702-707, 2011.

35. Yang C, Zheng S, Liu T, Liu Q, Dai F, Zhou J, Chen Y, Sheyhidin I and $\mathrm{Lu} \mathrm{X}$ : Down-regulated miR-26a promotes proliferation, migration, and invasion via negative regulation of MTDH in esophageal squamous cell carcinoma. FASEB J 31: 2114-2122, 2017.

36. Guan L, Ji D, Liang N, Li S and Sun B: Up-regulation of miR-10b-3p promotes the progression of hepatocellular carcinoma cells via targeting CMTM5. J Cell Mol Med 22: 3434-3441, 2018.

37. Salomonis N, Dexheimer PJ, Omberg L, Schroll R, Bush S, Huo J, Schriml L, Ho Sui S, Keddache M, Mayhew C, et al: Integrated genomic analysis of diverse induced pluripotent stem cells from the progenitor cell biology consortium. Stem Cell Reports 7: 110-125, 2016.

38. Mok SR, Mohan S, Grewal N, Elfant AB and Judge TA: A genetic database can be utilized to identify potential biomarkers for biphenotypic hepatocellular carcinoma-cholangiocarcinoma. J Gastrointest Oncol 7: 570-579, 2016.

39. Shi KQ, Lin Z, Chen XJ, Song M, Wang YQ, Cai YJ, Yang NB, Zheng MH, Dong JZ, Zhang L and Chen YP: Hepatocellular carcinoma associated microRNA expression signature: Integrated bioinformatics analysis, experimental validation and clinical significance. Oncotarget 6: 25093-25108, 2015.

40. Shen J, Wang A, Wang Q, Gurvich I, Siegel AB, Remotti H and Santella RM: Exploration of genome-wide circulating microRNA in hepatocellular carcinoma: MiR-483-5p as a potential biomarker. Cancer Epidemiol Biomarkers Prev 22: 2364-2373, 2013

41. Hung CH, Hu TH, Lu SN, Kuo FY, Chen CH, Wang JH, Huang CM, Lee CM, Lin CY, Yen YH and Chiu YC: Circulating microRNAs as biomarkers for diagnosis of early hepatocellular carcinoma associated with hepatitis B virus. Int J Cancer 138: 714-720, 2016

42. Liu M, Liu J, Wang L, Wu H, Zhou C, Zhu H, Xu N and Xie Y: Association of serum microRNA expression in hepatocellular carcinomas treated with transarterial chemoembolization and patient survival. Plos One 9: e109347, 2014. 
43. Chen CY, Chung IH, Tsai MM, Tseng YH, Chi HC, Tsai CY, Lin YH, Wang YC, Chen CP, Wu TI, et al: Thyroid hormone enhanced human hepatoma cell motility involves brain-specific serine protease 4 activation via ERK signaling. Mol Cancer 13: $162,2014$.

44. Wei L, Lian B, Zhang Y, Li W, Gu J, He X and Xie L: Application of microRNA and mRNA expression profiling on prognostic biomarker discovery for hepatocellular carcinoma. BMC Genomics 15 (Suppl 1): S13, 2014.

45. Lee YH, Andersen JB, Song HT, Judge AD, Seo D, Ishikawa T, Marquardt JU, Kitade M, Durkin ME, Raggi C, et al: Definition of ubiquitination modulator COP1 as a novel therapeutic target in human hepatocellular carcinoma. Cancer Res 70: 8264-8269, 2010.

46. Liao CJ, Chi HC, Tsai CY, Chen CD, Wu SM, Tseng YH, Lin YH, Chung IH, Chen CY, Lin SL, et al: A novel small-form NEDD4 regulates cell invasiveness and apoptosis to promote tumor metastasis. Oncotarget 6: 9341-9354, 2015.

47. Xu J, Zhou J, Li MS, Ng CF, Ng YK, Lai PB and Tsui SK Transcriptional regulation of the tumor suppressor FHL2 by p53 in human kidney and liver cells. PLoS One 9: e99359, 2014.

48. Martin-Mateos R, De Assuncao TM, Arab JP, Jalan-Sakrikar N, Yaqoob U, Greuter T, Verma VK, Mathison AJ, Cao S, Lomberk G, et al: Enhancer of zeste homologue 2 inhibition attenuates TGF- $\beta$ dependent hepatic stellate cell activation and liver fibrosis. Cell Mol Gastroenterol Hepatol 7: 197-209, 2019.

49. Sang L, Wang XM, Xu DY and Zhao WJ: Bioinformatics analysis of aberrantly methylated-differentially expressed genes and pathways in hepatocellular carcinoma. World J Gastroenterol 24: 2605-2616, 2018.

50. Honda M, Yamashita T, Yamashita T, Arai K, Sakai Y, Sakai A, Nakamura M, Mizukoshi E and Kaneko S: Peretinoin, an acyclic retinoid, improves the hepatic gene signature of chronic hepatitis $\mathrm{C}$ following curative therapy of hepatocellular carcinoma. BMC Cancer 13: 191, 2013.

51. Van Keuren-Jensen KR, Malenica I, Courtright AL, Ghaffari LT, Starr AP, Metpally RP, Beecroft TA, Carlson EW, Kiefer JA, Pockros PJ and Rakela J: MicroRNA changes in liver tissue associated with fibrosis progression in patients with hepatitis C. Liver Int 36: 334-343, 2016.

52. Leung WK, He M, Chan AW,Law PT and Wong N: Wnt $/ \beta$-Catenin activates MiR-183/96/182 expression in hepatocellular carcinoma that promotes cell invasion. Cancer Lett 362: 97-105, 2015.

53. Behnke M, Reimers M and Fisher R: The expression of embryonic liver development genes in hepatitis $\mathrm{C}$ induced cirrhosis and hepatocellular carcinoma. Cancers (Basel) 4: 945-968, 2012.

54. Karim S, Mirza Z, Chaudhary AG, Abuzenadah AM, Gari M and Al-Qahtani MH: Assessment of radiation induced therapeutic effect and cytotoxicity in cancer patients based on transcriptomic profiling. Int J Mol Sci 17: 250, 2016.

55. Schulze K and Zucman-Rossi J: Current issues on genomic heterogeneity in hepatocellular carcinoma and its implication in clinical practice. Hepat Oncol 2: 291-302, 2015.

56. Kan Z, Zheng H, Liu X, Li S, Barber TD, Gong Z, Gao H, Hao K, Willard MD, Xu J, et al: Whole-genome sequencing identifies recurrent mutations in hepatocellular carcinoma. Genome Res 23: 1422-1433, 2013.
57. Cao MQ, You AB, Zhu XD, Zhang W, Zhang YY, Zhang SZ, Zhang KW, Cai H, Shi WK, Li XL, et al: MiR-182-5p promotes hepatocellular carcinoma progression by repressing FOXO3a. J Hematol Oncol 11: 12, 2018

58. Wang Y, Lee AT, Ma JZ, Wang J, Ren J, Yang Y, Tantoso E, Li KB, Ooi LL, Tan P and Lee CG: Profiling microRNA expression in hepatocellular carcinoma reveals microRNA-224 up-regulation and apoptosis inhibitor-5 as a microRNA-224-specific target. J Biol Chem 283: 13205-13215, 2008.

59. Huang $\mathrm{H}$ and Tindall DJ: Dynamic FoxO transcription factors. J Cell Sci 120: 2479-2487, 2007.

60. Calvisi DF, Ladu S, Pinna F, Frau M, Tomasi ML, Sini M, Simile MM, Bonelli P, Muroni MR, Seddaiu MA, et al: SKP2 and CKS1 promote degradation of cell cycle regulators and are associated with hepatocellular carcinoma prognosis. Gastroenterology 137: 1816-1826.e1-10, 2009.

61. Dong T, Zhang Y, Chen Y, Liu P, An T, Zhang J, Yang H, Zhu W and Yang X: FOXO1 inhibits the invasion and metastasis of hepatocellular carcinoma by reversing ZEB2-induced epithelial-mesenchymal transition. Oncotarget 8: 1703-1713, 2017.

62. Shang YK, Li F, Zhang Y,Liu ZK, Wang ZL, Bian H and Chen ZN: Systems analysis of key genes and pathways in the progression of hepatocellular carcinoma. Medicine (Baltimore) 97: e10892, 2018.

63. Kitazawa S, Denda A, Tsutsumi M, Tsujiuchi T, Hasegawa K, Tamura K, Maruyama H and Konishi Y: Enhanced preneoplastic liver lesion development under 'selection pressure' conditions after administration of deoxycholic or lithocholic acid in the initiation phase in rats. Carcinogenesis 11: 1323-1328, 1990.

64. Tsuda H, Asamoto M, Kagawa M, Uwagawa S, Inoue K, Inui M and Ito N: Positive influence of dietary deoxycholic acid on development of pre-neoplastic lesions initiated by $\mathrm{N}$-methyl-N-nitrosourea in rat liver. Carcinogenesis 9: 1103-1105, 1988.

65. Yang F, Huang X, Yi T, Yen Y, Moore DD and Huang W: Spontaneous development of liver tumors in the absence of the bile acid receptor farnesoid X receptor. Cancer Res 67: 863-867, 2007.

66. Van Rooyen DM, Larter CZ, Haigh WG, Yeh MM, Ioannou G, Kuver R, Lee SP, Teoh NC and Farrell GC: Hepatic free cholesterol accumulates in obese, diabetic mice and causes nonalcoholic steatohepatitis. Gastroenterology 141: 1393-1403, 1403.e1-5, 2011.

67. Ioannou GN, Morrow OB, Connole ML and Lee SP: Association between dietary nutrient composition and the incidence of cirrhosis or liver cancer in the United States population. Hepatology 50: 175-184, 2009.

68. Matsuzawa N, Takamura T, Kurita S, Misu H, Ota T, Ando H, Yokoyama M,Honda M,Zen Y,Nakanuma Y, et al: Lipid-induced oxidative stress causes steatohepatitis in mice fed an atherogenic diet. Hepatology 46: 1392-1403, 2007.

This work is licensed under a Creative Commons Attribution-NonCommercial-NoDerivatives 4.0 International (CC BY-NC-ND 4.0) License. 